\title{
The modulatory properties of Astragalus membranaceus treatment on endometrial cancer: an integrated pharmacological method
}

\author{
Zhang Qianqian ${ }^{1}$, Huang Xianghua ${ }^{\text {Corresp. } 1}$ \\ ${ }^{1}$ Department of Gynecology, Hebei Medical University Second Affiliated Hospital, Shijiazhuang, China \\ Corresponding Author: Huang Xianghua \\ Email address: xianghuahuang0311@163.com
}

Astragalus membranaceus is a traditional Chinese medicine and has been used for adjuvant clinical therapy for a variety of cancers. However, the mechanism of its action on endometrial carcinoma is unclear. Based on the Gene Expression Omnibus (GEO) database, the Cancer Genome Atlas (TCGA) database, and the Traditional Chinese Medicine System Pharmacology Database $\left(\right.$ TCMSP $^{\mathrm{TM}}$ ), the drug and target compounds were initially screened to construct a common network module. Twenty active compounds in Astragalus membranaceus were successfully identified, which hit by 463 potential targets related to endometrial cancer. Eight of the more highly predictive compounds (such as Jaranol, Bifendate, Isorhamnetin, Calycosin, 7-0-methylisomucronulatol, Formononetin, Kaempferol, Quercetin) were involved in DNA integrity checkpoint, cyclin-dependent protein kinase holoenzyme complex, and histone kinase activity. Additionally, Gene Ontology (GO) and Kyoto Encyclopedia of Genes and Genomes (KEGG) pathway confirmed that Astragalus membranaceus might play a role in the treatment of endometrial cancer through p53 signalling pathway, transcriptional misregulation in cancer, and endometrial cancer signalling pathway. Drug-target-pathway networks were constructed using Cytoscape to provide a visual perspective. In addition, we verified that formononetin inhibited the proliferation of endometrial cancer cells through cell viability tests and clone formation tests. And qPCR and western blot found that formononetin exerts anti-cancer effects by promoting the expression of estrogen receptor beta (ER $\beta)$ and $p 53$. Based on a systematic network pharmacology approach, our works successfully predict the active ingredients and potential targets of Astragalus membranaceus for application to endometrial cancer and helps to illustrate mechanism of action on a comprehensive level. 
1 The modulatory properties of Astragalus membranaceus treatment on endometrial cancer:

2 an integrated pharmacological method

3 Qianqian Zhang ${ }^{1}$, Xianghua Huang 1

$4{ }^{1}$ Department of Gynecology, The Second Hospital of Hebei Medical University, 215 Heping

5 Road, Shijiazhuang, Hebei 050000, P. R. China

6

7 Corresponding author:

8 Xianghua Huang,

9 Department of Gynecology, The Second Hospital of Hebei Medical University, 215 Heping

10 Road, Shijiazhuang, Hebei 050000, P. R. China

11 Email address: xianghuahuang0311@163.com

12 ORCD: https://orcid.org/0000-0002-0104-4318

13

14

15

16

17

18

19

20

21

22

23

24

Peer) reviewing PDF | (2021:02:58138:2:0:NEW 17 Jul 2021) 


\section{ABSTRACT}

Astragalus membranaceus is a traditional Chinese medicine and has been used for adjuvant clinical therapy for a variety of cancers. However, the mechanism of its action on endometrial carcinoma is unclear. Based on the Gene Expression Omnibus (GEO) database, the Cancer Genome Atlas (TCGA) database, and the Traditional Chinese Medicine System Pharmacology Database (TCMSPTM), the drug and target compounds were initially screened to construct a common network module. Twenty active compounds in Astragalus membranaceus were successfully identified, which hit by 463 potential targets related to endometrial cancer. Eight of the more highly predictive compounds (such as Jaranol, Bifendate, Isorhamnetin, Calycosin, 7-O-methylisomucronulatol, Formononetin, Kaempferol, Quercetin) were involved in DNA integrity checkpoint, cyclin-dependent protein kinase holoenzyme complex, and histone kinase activity. Additionally, Gene Ontology (GO) and Kyoto Encyclopedia of Genes and Genomes (KEGG) pathway confirmed that Astragalus membranaceus might play a role in the treatment of endometrial cancer through p53 signalling pathway, transcriptional misregulation in cancer, and endometrial cancer signalling pathway. Drug-target-pathway networks were constructed using Cytoscape to provide a visual perspective. In addition, we verified that formononetin inhibited the proliferation of endometrial cancer cells through cell viability tests and clone formation tests. And qPCR and western blot found that formononetin exerts anti-cancer effects by promoting the expression of estrogen receptor beta $(\mathrm{ER} \beta)$ and $\mathrm{p} 53$. Based on a systematic network pharmacology approach, our works successfully predict the active ingredients and potential targets of Astragalus membranaceus for application to endometrial cancer and helps to illustrate mechanism of action on a comprehensive level.

Keywords: Astragalus membranaceus, network pharmacology, endometrial cancer, mechanism 
51 of action, target prediction.

\section{Abbreviations}

GEO: Gene Expression Omnibus, TCGA: The Cancer Genome Atlas, TCMSPTM: Encyclopedia of Genes and Genomes, EC: Endometrial Cancer, TCM: Traditional Chinese Medicine, TNBC: Triple-negative Breast Cancer, DBD: Danggui Buxue Decoction, MCC: Metastatic Colon Cancer, OB: Oral Bioavailability, DL Drug-Likeness, PPI: Protein-protein Interaction, DC: Degree, BC: Betweenness Centrality.

\section{INTRODUCTION}

61

62

The incidence and mortality of endometrial cancer (EC) has been increasing in recent years due to obesity, diabetes mellitus, hypertension, a continuous oestrogen supply unopposed by progesterone, infertility, nulliparity, and tamoxifen use (Wang, Zhou \& Wang, 2019). By 2020, there were an estimated 65,620 new cases and 12,590 deaths, making uterine cancer the fourth most common cancer in women and the fifth most common cause of cancer death (Siegel, Miller \& Jemal, 2020). Although our understanding of the biology of endometrial cancer and therapeutic strategies has dramatically progressed and improved, adjuvant treatment recommendations for this cancer remain complicated and controversial (Brooks et al., 2019). The treatment includes a combination of surgery, radiation therapy, and chemotherapy, including paclitaxel and cisplatin, but these treatments may not effectively improve the prognosis and produce unwanted side effects (Colombo et al., 2016; Fontanella et al., 2017; Webb et al., 2019). Therefore, there is a strong medical need to determine more effective and safer drugs to improve the prognosis and survival of EC patients.

4 Astragalus membranaceus, dried root extract, also known as Astragali radix, is first recorded in the "Shen Nong's Herbs" of the Han Dynasty 2000 years ago (Shan, Zheng \& Li, 
76

77

2019). So far, more than 200 compounds have been identified in Astragalus membranaceus, including saponins, polysaccharides, flavonoids, and some others. The diversity of constituents of Astragalus membranaceus is the material basis for its extensive pharmacological effects (Liao et al., 2017; Shan, Zheng \& Li, 2019; Xie et al., 2016). Accumulating pharmacological studies and clinical evidence centred on Astragalus membranaceus have reported anti-inflammatory, immunomodulatory, and anti-tumour effects, including in gynecologic oncology (Auyeung, Han \& Ko, 2016; Ong et al., 2019; Zhang et al., 2018). In cell models, astragaloside IV inhibits the invasion of cervical cancer by regulating various proteins on the surface of cervical cancer cells to induce autophagy (Xia, He \& Cai, 2020). In addition, a recent study on myeloid Graffi tumour showed that the saponins have time- and concentration-dependent anti-cell survival and proliferation pharmacological effects (Georgieva et al., 2021). Moreover, several studies have shown that Astragalus polysaccharides inhibit the proliferation and metastasis of ovarian cancer (Guo et al., 2020a), breast cancer (Li et al., 2019), and prostate cancer (Guo et al., 2020b) through immune regulation, autophagy regulation, anti-oxidation and regulation of inflammation. However, few studies have investigated the effects of Astragalus membranaceus on EC or the biotargets and molecular mechanisms involved in the effects of Astragalus membranaceus.

Network pharmacology is an emerging and novel method to discover the basis of pharmacodynamic substances and explore their molecular mechanisms of action (Sidders et al., 2018). In particular, TCM network pharmacology focuses on the holistic and systematic nature of the interactions between TCM compounds, targets and diseases (Zhang et al., 2017). Liu et al. used a comprehensive network pharmacology method to identify the key compounds and targets of Astragalus membranaceus for triple-negative breast cancer (TNBC). In vitro experiments showed that the effective compounds of Astragalus membranaceus might inhibit the invasion of TNBC through the PIK3CG/AKT/BCL2 pathway (Liu et al., 2019a). Furthermore, using the same method, Feng et al. identified 28 active compounds and 61 common targets of Danggui Buxue Decoction (DBD) on metastatic colon cancer (MCC), providing a scientific reference for the treatment of MCC in traditional Chinese medicine (Feng et al., 2021). Therefore, TCM 
103

104

105

106

107

108

109

110

111

112

113

114

115

116

117

118

119

120

121

122

123

124

125

126

127

systems pharmacology may be a promising strategy for understanding the pharmacological targets and mechanisms of Astragalus membranaceus in EC.

In the present study, we aimed to use a network pharmacology approach to establish the effects of Astragalus membranaceus on EC and predict core targets and biological functions, pathways, and mechanisms of action. Notably, this is the first comprehensive study using multiple methods to clarify the antitumour efficacy substances and mechanism(s) of action of Astragalus membranaceus on endometrial cancer.

\section{MATERIALS AND METHODS}

\section{Data mining based on the GEO and TCGA database}

The gene expression profile GSE63678 dataset (Pappa et al., 2015) was downloaded from the GEO database (https://www.ncbi.nlm.nih.gov/geo/). The platform for GSE63678 is GPL571 ([HG-U133A_2] Affymetrix Human Genome U133A 2.0 Array), including 5 normal tissues and 7 tumour tissues. The probes were converted into the corresponding gene site according to the annotation information on the platform. All gene expression data were subjected to $\log 2$ transformation.

The Cancer Genome Atlas (TCGA) is a free and comprehensive database that includes large clusters of human cancer genome sequencing data (https://Cancergenome.nih.gov/). The standard for data inclusion in our study was that the sample size must consist of both cancer and normal tissues, and that the number of normal tissue controls must be greater than or equal to three. In this study, we obtained 587 cases of gene expression data (35 cases of normal endometrial samples \& 552 cases of EC samples) from TCGA official website for the Uterine Corpus Endometrial Carcinoma projects (UCEC). Before further analysis and processing, RNA sequencing data standardization was carried out in full accordance with TCGA's release guidelines.

Peer) reviewing PDF | (2021:02:58138:2:0:NEW 17 Jul 2021) 


\section{Identification of DEGs in EC}

130

131

132

The "affy" R language package was applied to complete the $\log 2$ transformation, quantile normalization and median polish algorithm summarization. The probes were annotated by the Affymetrix annotation files. Microarray quality was evaluated by sample clustering based on the distance between different samples in Pearson's correlation matrices (Liu et al., 2019b). The limma package was used to detect DEGs between the normal and EC tissues (Zhang et al., 2019). Screening criteria of $\mid \log 2$-fold-change (FC) $\mid \geq 1$ and P-value $<0.05$ were considered indicators of statistical significance in the identification of robust DEGs. In the same way, we used the R software (version: x64 4.1.0) and the "edgeR" package to screen the DEGs in the TCGA dataset. The screening criteria were: $\mid \log 2$-fold-change $(\mathrm{FC}) \mid \geq 1$ and P-value $<0.05$. Subsequently, we took the intersected DEGs of the two datasets by the Venn package in the R software.

\section{Chemical compound database building}

The Traditional Chinese Medicine System Pharmacology Database (Ru et al., 2014) (TCMSPTM, http://lsp.nwu.edu.cn/tcmsp.php) is a resource of systems pharmacology for TCMs and related compounds. We collected the data on Astragalus membranaceus active compounds using the ADME (absorption, distribution, metabolism, and excretion) filter method, whose main parameters included oral bioavailability (OB) and drug-likeness (DL) (Zhang et al., 2016). OB of $\geq 30 \%$ and DL of $\geq 0.18$ were the threshold values for ADME evaluation systems. When herbal compounds met both criteria $(\mathrm{OB} \geq 30 \%$, $\mathrm{DL} \geq 0.18)$, they were selected as candidates for further analysis. The chemical name "Astragalus membranaceus" was entered into the search box, and its pharmacokinetic properties were studied at the molecular level.

\section{Disease targets database building and network construction}


We introduced Astragalus membranaceus-regulated target genes and EC-related target

154

155

156

157

158

159

160

161

162

163

164

165

166

167

168

169

170

171

172

173

174

175

176

177

genes into the Perl script to get overlapping targets and active compounds, which were the potential targets of Astragalus membranaceus against EC. Subsequently, these overlapping parts were introduced into Cytoscape 3.7.1 software (http://www.cytoscape.org/) for network construction and visualization (Holmås et al., 2019). In the network, "node" represents the corresponding compound and target genes, and "edge" represents the relationship between the compound and the target genes.

\section{Protein-protein interaction (PPI) network construction and topology analysis}

We introduced the overlapping gene targets into Cytoscape software for PPI analysis. The BisoGenet 3.0.0 package in the Cytoscape software was used to construct a protein interaction network (Martin et al., 2010). Then, we used the "CytoNCA" plugin of the Cytoscape software for topology analysis (Tang et al., 2015). For each node in the interaction network, "degree (DC)" and "betweenness centrality (BC)" were selected to assess its topological features. DC represents the number of node links and reflects how often a node interacts with other nodes (Missiuro et al., 2009). BC is defined as the degree to which a node is located on a path between other nodes (Raman, Damaraju \& Joshi, 2014). The original PPI network was screened twice according to the values of DC and BC to obtain the core PPI network.

\section{Gene function and pathway enrichment analysis}

The "ggplot2" package in R software for Gene Ontology (GO) and Kyoto Encyclopedia of Genes and Genomes (KEGG) analysis (Maag, 2018), to obtain the biological process, cellular compounds, molecular function, and critical signalling pathways (The Gene Ontology Consortium, 2017). In addition, to better understand the complex relationship between core biological pathways and hub co-targets associated with the Astragalus 
178 179

180

181

182

183

membranaceus, we used Cytoscape 3.7.1 (http://www.cytoscape.org/) to build and analyse networks.

\section{Reagents and Cells}

Formononetin $\left(\mathrm{C}_{16} \mathrm{H}_{12} \mathrm{O}_{4}, 98 \%\right.$ purity verified by high-performance liquid chromatography) was obtained from Phytomarker Ltd., Tianjin, China. Formononetin was dissolved in dimethyl sulfoxide (DMSO) as a stock solution of $100 \mathrm{mM}$. The $40 \mu \mathrm{M}$ and $80 \mu \mathrm{M}$ concentrations of formononetin in experiments were obtained by diluting the medium to a final concentration of DMSO $<0.1 \%(\mathrm{vol} / \mathrm{vol})$. Cells treated with $0.1 \%$ DMSO served as a control. The solution was stored at $4^{\circ} \mathrm{C}$ for further use.

Human endometrial cancer cell lines (Ishikawa, HEC-1A, and HEC-251) were purchased from the Institute of Biochemistry and Cell Biology of the Chinese Academy of Sciences (Shanghai, PR China). All cell lines were maintained in Dulbecco's modified Eagle medium (DMEM), or DMEM/F12 supplemented with 10\% heat-inactivated fetal bovine serum (FBS) and kept at a $37^{\circ} \mathrm{C}, 5 \% \mathrm{CO}_{2}$ incubator. Cell lines were routinely checked for mycoplasma contamination.

\section{Cell viability assay}

Cell proliferation was performed by a Cell Counting Kit-8 (CCK-8) (Meilun Biotechnology Co., Ltd., MA0218-5) according to the manufacturer's protocol. Briefly, the Ishikawa, HEC-1A, and HEC-251 cell lines were trypsinized and seeded into 96-well plates at a density of $1 \times 10^{4}$ each well. After incubating overnight at $37^{\circ} \mathrm{C}$, the cells were treated with a concentration of $40 \mu \mathrm{M}$ and $80 \mu \mathrm{M}$ of formononetin for $24 \mathrm{~h}$. The absorbance of each well was determined at 490 nm under a Smart Microplate Reader (USCNK, Wuhan). 
203

204

205

206

207

208

209

210

211

212

213

214

215

216

217

218

219

220

221

222

223

224

225

226

227

\section{Colony formation assay}

The effect of formononetin on the colony-forming ability of endometrial cancer cells was evaluated by colony formation assay. The Ishikawa, HEC-1A, and HEC-251 cell lines were seeded into 6-well plates at the concentration of 400 cells per well. After incubating for $24 \mathrm{~h}$ at $37^{\circ} \mathrm{C}$, the cells were incubated with formononetin $(40 \mu \mathrm{M}$ and $80 \mu \mathrm{M})$. Three days later, most single colonies contain more than 50 cells. Cells were harvested and rinsed three times with distilled water, and images were obtained by a microscope.

\section{RNA extraction and qPCR assays}

After exposure to formononetin $(40 \mu \mathrm{M}$ and $80 \mu \mathrm{M})$ for $48 \mathrm{~h}$, the Ishikawa, HEC-1A, and HEC-251 cells were harvested for RNA isolation by using a TRIZOL®reagent (TaKaRa, 9109). Then, cDNA was subsequently synthesized using $10 \mathrm{ng}$ of RNA under standard conditions for the iScript cDNA Synthesis Kit (Fermentas Inc., USA). Quantitative polymerase chain reaction (qPCR) was performed by SYBR Premix Ex Taq II (Takara, Japan). The $2^{-\Delta \Delta \mathrm{Ct}}$ method was used to calculate the relative expression. The gene primers were listed as follows: ER $\beta$ : 5'AATGGGGTTCTCTCCTGT' (sense) and 5'-AGCCCAAAGTATCCCTGAC-3' (antisense); p53: 5'-CAGTAGTCAAGTAGTAACCCCTGCCTTGCACAG-3' (sense) and 5'-CATGTATTACTGTGCAAGGCAGGGGTTACTACTT-3' (antisense); Actin: 5'CATGTACGTTGCTATCCAGGC-3' (forward) and 5'-CTCCTTAATGTCACGCACGAT-3' (reverse).

\section{Western blotting}

After 48 hours of incubation with the designated concentration of formononetin, the Ishikawa, HEC-1A, and HEC-251 cells were lysed in RIPA buffer. The quantified protein was separated by $10 \%$ SDS-PAGE and subsequently transferred to polyvinylidene difluoride (PVDF) 
228

229

230

231

232

233

234

235

236

237

238

239

240

241

242

243

244

245

246

247

248

249

250

251

252

membranes (Bio-Rad, USA). Then the membrane was sealed with 5\% nonfat milk and incubated with primary antibodies including ER $\beta$ (1:2500; Proteintech Group, Inc. Wuhan, China), p53 (1:800; bsm-33058M; Beijing Bioss Biological Co., Ltd, Beijing, China), and $\beta$-actin (ABclonal, Inc. Wuhan, China) for $2 \mathrm{~h}$ at room temperature. After three washes with the TBST solution, the samples were incubated with the appropriate secondary antibodies for $2 \mathrm{~h}$. The blots were visualized with an electro-chemiluminescence (ECL) reagent kit (Beyotime, China). Image J software (National Institutes of Health, Bethesda, MD, USA) was used to quantify each protein band intensity, and the $\beta$-actin was used as an internal reference.

\section{Statistical analysis}

The data were presented as the mean \pm standard deviation (SD). The SPSS 25.0 software (SPSS, Chicago, IL) was used for statistical analysis using one-way ANOVA. All experiments were carried out at least three times, and a $\mathrm{P}<0.05$ was considered significant.

\section{RESULTS}

\section{Endometrial cancer-related targets}

Based on the DEG selection criteria of $\mid \log 2$-fold-change $(\mathrm{FC}) \mid \geq 1$ and P-value $<0.05$, a total of 360 DEGs, including 204 upregulated DEGs and 156 downregulated DEGs, were identified between the normal and EC tissues after consolidation and normalization of the microarray data from the GSE63678 dataset (Figure 1). In addition, a total of 11531 DEGs were obtained from the TCGA database. Cluster analysis (Figure 2) revealed 7727 upregulated genes and 3804 downregulated genes. The Venn package was used to screen the intersecting DEGs from both databases and generate the Venn map (Figure 3). Finally, 268 EC-related DEGs with high reliability were obtained (Supplementary file, Table S1 for details).

Peer] reviewing PDF | (2021:02:58138:2:0:NEW 17 Jul 2021) 
253

254

255

256

257

258

259

260

261

262

263

264

265

266

267

268

269

270

271

272

273

274

275

276

\section{Active compounds in Astragalus membranaceus and the targets}

\section{Disease targets database building and network construction}

A total of 10 overlapping genes and 8 overlapping potentially active compounds were screened as all targets for Astragalus membranaceus treatment of EC (Table 2). As shown in Figure 4, the compound-target network consisted of 18 nodes and 19 edges. The top 3 compounds of Astragalus membranaceus with the highest values of both $\mathrm{DC}$ and $\mathrm{BC}$, in descending order by DC, were kaempferol (MOL000422), formononetin (MOL000392), and quercetin (MOL000098), and they will likely play a more significant role in treating EC.

\section{PPI network construction and topology analysis}

We obtained the core PPI network after the original PPI network screened according to DC and BC (Figure 5). The core PPI network consisted of 48 nodes and 528 edges. In other words, the core PPI network contained 48 compounds and target genes, and there were 528 relationships between them (Figure 5C). Genes, namely, TP53, NPM1, YWHAZ, HSP90AA1, and BRCA1 with the highest DC and BC values, are likely the critical targets in EC. membranaceus and EC 
GO analysis (Figure 6A-C) and pathway enrichment analysis (Figure 6D) were performed

278

279

280

281

282

283

284

285

286

287

288

289

290

291

292

293

294

295

296

297

298

299

300

301

302

to clarify the biological functions of these 10 target genes. The screening condition was $\mathrm{P}<0.05$ and $\mathrm{Q}<0.05$. The BP category of the GO analysis results showed that the target genes were markedly associated with histone phosphorylation, DNA integrity checkpoint, and signal transduction involved in DNA integrity checkpoint. For the CC category, the target genes were markedly related to the cyclin-dependent protein kinase holoenzyme complex, condensed chromosome and serine/threonine protein kinase complex. The MF category was significantly enriched in histone kinase activity, heat shock protein binding, and Hsp70 protein binding. The KEGG signalling pathway analysis showed that the target genes played pivotal roles in the following pathways: 'p53 signalling pathway', 'Transcriptional misregulation in cancer', 'EGFR tyrosine kinase inhibitor resistance', and 'Endometrial cancer'.

In addition, based on the target and pathway analyses, an entire compound, target and disease network was constructed by Cytoscape (v 3.7.1). As shown in Figure 7, this compound, target, and disease interaction network had 23 nodes and 43 edges. Red inverted triangles and orange rectangles correspond to the pathway and target genes, respectively.

\section{Anti-proliferation effect of formononetin on endometrial cancer cells}

Formononetin, as an immunomodulator, has been widely used for centuries. Formononetin's anti-tumour effects such as inhibiting cell proliferation, inducing cell cycle arrest and apoptosis have been confirmed in bladder cancer(Wu et al., 2017), breast cancer(Xin et al., 2019), and ovarian cancer (Park et al., 2018). However, the role of formononetin in endometrial cancer cells remains to be explored. Therefore, we choose formononetin for verification.

The effect of formononetin on the proliferation of endometrial cancer cell lines was tested by cell viability assay and clone formation assay. As shown in Figure 8, formononetin significantly inhibited the proliferation rate of endometrial cancer cell lines compared with the control group (all $\mathrm{P}<0.05$ relative to the control group). In addition, compared with $40 \mu \mathrm{M}$ of 
303

304

305

306

307

308

309

310

311

312

313

314

315

316

317

318

319

320

321

322

323

324

325

326

327

formononetin, higher concentrations inhibited the cells more strongly (all $\mathrm{P}<0.05$ relative to the $40 \mu \mathrm{M}$ group). These results indicate that formononetin may inhibit cell proliferation in a dosedependent manner.

\section{The effect of formononetin on the expression of ERß and p53}

ER $\beta$ has been widely reported to play an important role in inhibiting cell proliferation. Furthermore, KEGG pathway analysis showed that Astragalus membranaceus could act on EC through the p53 signalling pathway. In order to explore how formononetin exerts an anti-tumor effect, we explore the differences in ER $\beta$ and p53 between different groups. In different groups of endometrial cancer cell lines, the expression of ER $\beta$ and p53 in the cells treated with formononetin was significantly increased, as expected (all $\mathrm{P}<0.05$ relative to the control group); In addition, the higher the concentration of formononetin, the more the expression of ER $\beta$ and p53 (all $\mathrm{P}<0.05$ relative to the $40 \mu \mathrm{M}$ group; Figure 9A and B). To add more evidence, we performed Western blots on ER $\beta$ and p53 using the same cells. Indeed, formononetin can promote the expression of ER $\beta$ and p53 in a dose-dependent manner (Figure 9C).

\section{DISCUSSION}

Although our understanding of endometrial cancer has been profoundly advanced in recent years, the occurrence and progression of cancer is an intricate process; therefore, endometrial cancer may not effectively respond to a single therapeutic intervention measure (Arend et al., 2018; Urick \& Bell, 2019). As a traditional Chinese herb, Astragalus membranaceus has been widely used clinically for the treatment of cancer, including EC. In clinical practice, Astragalus membranaceus injection can increase blood cells and immunoglobulins in patients with EC who were undergoing chemotherapy, and reduce bone marrow suppression (Xiyao, Chao \& Lihong, 2019). Another clinical study showed that the expression of neutrophil gelatinase-related 
328 lipocalin and its receptor in the serum of chemotherapy patients who took Astragalus injection 329 were reduced, and the recurrence rate within 3 years was significantly lower than that of EC 330 patients who did not take (Yufang \& Yan, 2019). Preclinical studies have shown that Astragalus 331 injection can promote the expression of E-cadherin and reduce the expression of $\beta$-catenin in EC 332 cells, thereby inhibiting the WNT signalling pathway to exert anti-cancer effects (Xiaoming et al., 333 2016). In addition, studies by Gao et al. have shown that Astragalus injection can effectively 334 reduce the levels of carcinoembryonic antigen 125 (CA125) and human epididymal secretory 335 protein 4 (HE4) in the serum of EC patients, and reduce the toxicity of chemotherapy (Gao, 336 2018). Furthermore, regarding the Chinese medicine SH003, whose main ingredient was 337 Astragalus membranaceus, the phase I clinical study on the safety of patients with solid tumours 338 was also underway (Cheon \& Ko, 2020). In the present study, we used a network pharmacological approach to explore the material basis and molecular mechanisms of Astragalus membranaceus for the treatment of EC.

Dong et al. studied Astragalus membranaceus against laryngeal cancer through network pharmacology, showing that quercetin, rutin, and chlorogenic acid in Astragalus membranaceus are the main compounds that exert anti-cancer effects (Dong et al., 2020). Research by Liu et al. showed that 7-O-methyl iso-mucronulatol, quercetin, 3,9-di-O-methylnissolin, isorhamnetin, and formononetin in Astragalus membranaceus could activate the immune system to fight diseases by regulating the $\mathrm{T}$ cell immune response, the tropism of macrophages and the production of antimicrobial peptides (Liu et al., 2019c). In the current study, we obtained 8 active compounds and 10 compound-disease targets in total. According to the analysis of the network, the interaction between these effective active compound and target genes indicates that Astragalus membranaceus acts on EC in a multi-compound and multitarget manner. The active compounds 351 of Astragalus membranaceus with the highest DC values were kaempferol (MOL000422), 352 quercetin (MOL000098), and formononetin (MOL000392). In addition, the top 5 target genes 
354 355

356

357

358

359

360

361

362

363

364

365

366

367

368

369

370

371

372

373

374

375

376

377

378

379

380

speculated that these targets might be key targets for Astragalus membranaceus-based treatments of EC.

Kaempferol represents one of the most common glycoside forms of aglycone flavonoids ( $\mathrm{Li}$ et al., 2015). Previous studies have shown that kaempferol and its glycosylated derivatives have anti-inflammatory, anti-diabetic, anti-oxidant, and anti-tumour effects and have anti-cancer activity (Imran et al., 2019). High concentrations of kaempferol have a strong antitumour effect by promoting apoptosis and downregulating signalling pathways, inositol 3-kinase $(\mathrm{PI} 3 \mathrm{~K}) /$ protein kinase B (AKT) action and the epithelial-mesenchymal transition (EMT) (Chen \& Chen, 2013; Wang et al., 2019). An investigation has shown that the inhibitory effect of kaempferol on endometrial cancer is induced by apoptosis, G2/M phase cell cycle arrest, inhibition of cell invasion, and the upregulation of the m-TOR/PI3K signalling pathway (Chuwa et al., 2018; Lei et al., 2019). Quercetin is one of the most important flavonoids in the human diet (Lin et al., 2017). Quercetin has attracted considerable attention because of its amazing anticancer properties, such as cell signalling, pro-apoptosis, anti-proliferative and anti-oxidant effects, and growth inhibition (Farooqi et al., 2018). In fact, the ability of quercetin to inhibit the action of multiple enzymes involved in cell proliferation and multiple signal transduction pathways in endometrial cancer has been established (Kaneuchi et al., 2003; Scambia et al., 1992; Wang et al., 2009).

Formononetin is widely known as a phytoestrogen that can replace oestrogen. Relevant literature and data have shown that formononetin can inhibit various cancer cells, especially cancers of the reproductive system, including breast cancer, ovarian cancer and cervical cancer (Zhang et al., 2018; Zhang, Chen \& Zhang, 2019). Chen et al. found that formononetin inhibited the proliferation of ER-positive breast cancer cells and triggered apoptosis in a time and dosedependent manner by promoting the increase of ER- $\beta$ expression (Chen et al., 2013). Moreover, studies have shown that formononetin inhibits neuroinflammation by promoting the expression of ER- $\beta$ (El-Bakoush \& Olajide, 2018). Our network pharmacological analysis showed that formononetin, as a crucial active ingredient in Astragalus membranaceus, also played a powerful 
381

382

383

384

385

386

387

388

389

390

391

392

393

394

395

396

397

398

399

400

401

402

403

404

405

406

anti-cancer effect in the treatment of endometrial cancer. In vitro studies showed that formononetin inhibited the proliferation of endometrial cancer by promoting the expression of ER $\beta$ and thus exerted an anti-cancer effect. This result was consistent with previous research. The potential mechanism for the formononetin-ER $\beta$ axis to exert anti-cancer effects may be that formononetin acts on microRNA-375, RASD1, or ER $\alpha$ to form a negative feedback loop (Chen et al., 2018), but this requires further verification.

The target protein interaction network (PPI) analysis in our study revealed that the main target proteins of Astragalus membranaceus active compounds in treating EC are TP53, NPM1, YWHAZ, HSP90AA1, and BRCA1. Extensive research and data indicated that these target proteins were closely related to multiple cancer signalling pathways (Bian et al., 2018; Dimitrakopoulos et al., 2018; Repana et al., 2019; Suen et al., 2019; Tamura et al., 2015). TP53 was a tumour suppressor gene which could induce cell cycle arrest, apoptosis, ageing, DNA repair or metabolic changes (Stine et al., 2015). Mutations in this gene had been implicated in a variety of human cancers, including EC (Cancer Genome Atlas Research Network et al., 2013). In vitro studies showed that formononetin inhibited the proliferation of endometrial cancer by promoting the expression of $\mathrm{p} 53$ and thus exerted an anti-cancer effect. A recent study showed that the plant extract methoxyeugenol inhibits the proliferation of endometrial cancer cells by activating the p53/p21 signalling pathway (Costa et al., 2021).

GO function enrichment analysis showed that these co-targets were mainly in DNA damage, cell cycle, and metabolism, which were closely related to cell canceration. The KEGG gene network revealed that targets CHEK1, BAX, CDK1, CCNB1, and CCNA2 were associated with some important pathways related to cancer metabolisms, such as the p53 signalling pathway, cell cycle, cellular senescence, transcriptional misregulation in cancer, and endometrial cancer pathway. As shown in the endometrial cancer pathway (hsa05213), both BAX and BIRC5 are Astragalus membranaceus targets that can inhibit tumour proliferation (Huo et al., 2019; Sakuragi et al., 2002). These results suggest that Astragalus membranaceus can effectively cure 
407

408

409

410

411

412

413

414

415

416

417

418

419

420

421

422

423

424

425

426

427

428

429

endometrial cancer and help patients recover from this disease because it is mainly involved in cancer-related metabolism pathways.

\section{CONCLUSIONS}

This study is the first to investigate the effects and mechanisms of Astragalus membranaceus against endometrial cancer by conducting network pharmacological analysis and experimental verification. The network pharmacology analysis findings showed that kaempferol, quercetin, and formononetin from Astragalus membranaceus exert anti-endometrial cancer pharmacological effects by regulating multiple targets and pathways. The experimental validation results suggested that formononetin may play an anti-cancer effect by up-regulating the expression of ER $\beta$ and p53. The enrichment analysis of GO and KEGG showed that the anticancer effect of Astragalus membranaceus might be related to the regulation of cell cycle, metabolism and some important cancer-related pathways. These findings provide a scientific basis for elucidating the mechanism of action of Astragalus membranaceus in the treatment of endometrial cancer. However, since the study was based on data mining and experimental verification, further clinical validation studies should be conducted to determine the role of Astragalus membranaceus in endometrial cancer.

\section{Acknowledgements}

This work was supported by the innovation ability of Hebei Obstetrics and Gynecology Clinical Medicine Research Center is improved (20577705D).

\section{Ethical approval}



applicable.

432

Declaration of conflicting interest

434

The authors declare that there is no conflict of interest.

435

436

\section{Data Availability}

437

438

439

440

441

442

443

444

445

446

447

448

449

450

451

452

The following information was supplied regarding data availability:

Raw data is available in the Supplementary Files.

The microarray-based expression data of EC patients were available at Gene Expression Omnibus: GSE63678.

The expression profile were all from the level 3 data available in the TCGA database (https://portal.gdc.cancer.gov/repository). Search terms: "Primary Site" IS "Uterus" AND "Project Id" IS “TCGA-UCEC” AND "Workflow Type” IS "HTSeq-FPKM" AND "Data Category” IS “TranscriptomeProfiling” AND “Data Type” IS “Gene Expression Quantification.”

\section{REFERENCES}

Arend RC, Jones BA, Martinez A, Goodfellow P. 2018. Endometrial cancer: Molecular markers and management of advanced stage disease. Gynecologic oncology 150:569-580 DOI 10.1016/j.ygyno.2018.05.015. 
Auyeung KK, Han QB, Ko JK. 2016. Astragalus membranaceus: A Review of its Protection Against Inflammation and Gastrointestinal Cancers. The American journal of Chinese medicine 44:1-22 DOI 10.1142/S0192415X16500014.

Bian X, Gao J, Luo F, Rui C, Zheng T, Wang D, Wang Y, Roberts TM, Liu P, Zhao JJ, Cheng H. 2018. PTEN deficiency sensitizes endometrioid endometrial cancer to compound PARP-PI3K inhibition but not PARP inhibition as monotherapy. Oncogene 37:341-351 DOI 10.1038/onc.2017.326.

Brooks RA, Fleming GF, Lastra RR, Lee NK, Moroney JW, Son CH, Tatebe K, Veneris JL. 2019. Current recommendations and recent progress in endometrial cancer. $C A$ : a cancer journal for clinicians 69:258-279 DOI 10.3322/caac.21561.

Chen AY, Chen YC. 2013. A review of the dietary flavonoid, kaempferol on human health and cancer chemoprevention. Food chemistry 138:2099-2107 DOI 10.1016/j.foodchem.2012.11.139.

Chen J, Zhang X, Wang Y, Ye Y, Huang Z. 2018. Differential ability of formononetin to stimulate proliferation of endothelial cells and breast cancer cells via a feedback loop involving MicroRNA-375, RASD1, and ERa. Molecular carcinogenesis 57:817-830 DOI 10.1002/mc. 22531 .

Chen J, Zhao X, Ye Y, Wang Y, Tian J. 2013. Estrogen receptor beta-mediated proliferative inhibition and apoptosis in human breast cancer by calycosin and formononetin. Cellular physiology and biochemistry : international journal of experimental cellular physiology, biochemistry, and pharmacology 32:1790-1797 DOI 10.1159/000356612.

Cheon C, Ko SG. 2020. A Phase I Study to Evaluate the Safety of the Herbal Medicine SH003 in Patients With Solid Cancer. Integrative cancer therapies 19:1534735420911442 DOI $10.1177 / 1534735420911442$.

Chuwa AH, Sone K, Oda K, Tanikawa M, Kukita A, Kojima M, Oki S, Fukuda T, Takeuchi M, Miyasaka A, Kashiyama T, Ikeda Y, Nagasaka K, Mori-Uchino M, Matsumoto Y, Wada-Hiraike O, Kuramoto H, Kawana K, Osuga Y, Fujii T. 2018. Kaempferol, a natural dietary flavonoid, suppresses $17 \beta$-estradiol-induced survivin expression and causes apoptotic cell death in endometrial cancer. Oncology letters 16:6195-6201 DOI 10.3892/ol.2018.9340.

Cancer Genome Atlas Research Network, Kandoth C, Schultz N, Cherniack AD, Akbani R, Liu Y, Shen H, Robertson AG, Pashtan I, Shen R, Benz CC, Yau C, Laird PW, Ding L, Zhang W, Mills GB, Kucherlapati R, Mardis ER, Levine DA. 2013. Integrated genomic characterization of endometrial carcinoma. Nature 497:67-73 DOI 10.1038/nature12113.

The Gene Ontology Consortium. 2017. Expansion of the Gene Ontology knowledgebase and 
resources. Nucleic acids research 45:D331-331D338 DOI 10.1093/nar/gkw1108.

490

491

492

493

494

495

496

497

498

499

500

501

502

503

504

505

506

507

508

509

510

511

512

513

514

515

516

517

518

519

520

521

522

523

524

Colombo N, Creutzberg C, Amant F, Bosse T, González-Martín A, Ledermann J, Marth C, Nout R, Querleu D, Mirza MR, Sessa C, ESMO-ESGO-ESTRO Endometrial Consensus Conference Working Group. 2016. ESMO-ESGO-ESTRO Consensus Conference on Endometrial Cancer: diagnosis, treatment and follow-up. Annals of oncology : official journal of the European Society for Medical Oncology 27:16-41 DOI 10.1093/annonc/mdv484.

Costa BP, Nassr MT, Diz FM, Fernandes K, Antunes GL, Grun LK, Barbé-Tuana FM, Nunes FB, Branchini G, de Oliveira JR. 2021. Methoxyeugenol regulates the p53/p21 pathway and suppresses human endometrial cancer cell proliferation. Journal of ethnopharmacology 267:113645 DOI 10.1016/j.jep.2020.113645.

Dimitrakopoulos C, Hindupur SK, Häfliger L, Behr J, Montazeri H, Hall MN, Beerenwinkel N. 2018. Network-based integration of multi-omics data for prioritizing cancer genes. Bioinformatics 34:2441-2448 DOI 10.1093/bioinformatics/bty148.

Dong KF, Huo MQ, Sun HY, Li TK, Li D. 2020. Mechanism of Astragalus membranaceus in the treatment of laryngeal cancer based on gene co-expression network and molecular docking. Scientific reports 10:11184 DOI 10.1038/s41598-020-68093-0.

El-Bakoush A, Olajide OA. 2018. Formononetin inhibits neuroinflammation and increases estrogen receptor beta (ER $\beta)$ protein expression in BV2 microglia. International immunopharmacology 61:325-337 DOI 10.1016/j.intimp.2018.06.016.

Farooqi AA, Jabeen S, Attar R, Yaylim I, Xu B. 2018. Quercetin-mediated regulation of signal transduction cascades and microRNAs: Natural weapon against cancer. Journal of cellular biochemistry 119:9664-9674 DOI 10.1002/jcb.27488.

Feng SH, Zhao B, Zhan X, Motanyane R, Wang SM, Li A. 2021. Danggui Buxue Decoction in the Treatment of Metastatic Colon Cancer: Network Pharmacology Analysis and Experimental Validation. Drug design, development and therapy 15:705-720 DOI 10.2147/DDDT.S293046.

Fontanella C, Barcellini A, Vitale MG, Lepori S, Maltese G, Tripodi E, Cerrotta A, Martinelli F, Andreetta C, Bogani G, Ditto A, Signorelli M, Scaffa C, Sacco C, Raspagliesi F, Lorusso D. 2017. Is chemotherapy worthwhile in patients with high-risk, lymph node negative, FIGO stage 1, endometrial cancer. Annals of oncology : official journal of the European Society for Medical Oncology 28 Suppl 6:vi70 DOI 10.1093/annonc/mdx429.002.

Gao G. 2018. Effect of Huangqi injection on the clinical effect of endometrial carcinoma and the effect of the serum CA125 and HE4 on the tumor markers. Shaanxi Journal of Traditional Chinese Medicine 39:762-765 DOI 10.3969/j.issn.1000-7369.2018.06.024. 
525 Georgieva A, Popov G, Shkondrov A, Toshkova R, Krasteva I, Kondeva-Burdina M,

526

527

528

529

530

531

532

533

534

535

536

537

538

539

540

541

542

543

544

545

546

547

548

549

550

551

552

553

554

555

556

557

558

559

560

Manov V. 2021. Antiproliferative and antitumour activity of saponins from Astragalus glycyphyllos on myeloid Graffi tumour. Journal of ethnopharmacology 267:113519 DOI 10.1016/j.jep.2020.113519.

Guo S, Ma B, Jiang X, Li X, Jia Y. 2020b. Astragalus Polysaccharides Inhibits Tumorigenesis and Lipid Metabolism Through miR-138-5p/SIRT1/SREBP1 Pathway in Prostate Cancer. Frontiers in pharmacology 11:598 DOI 10.3389/fphar.2020.00598.

Guo Y, Zhang Z, Wang Z, Liu G, Liu Y, Wang H. 2020a. Astragalus polysaccharides inhibit ovarian cancer cell growth via microRNA-27a/FBXW7 signaling pathway. Bioscience reports 40: DOI 10.1042/BSR20193396.

Holmås S, Puig RR, Acencio ML, Mironov V, Kuiper M. 2019. The Cytoscape BioGateway App: explorative network building from the BioGateway triple store. Bioinformatics DOI 10.1093/bioinformatics/btz835.

Huo X, Sun H, Liu Q, Ma X, Peng P, Yu M, Zhang Y, Cao D, Shen K. 2019. Clinical and Expression Significance of AKT1 by Co-expression Network Analysis in Endometrial Cancer. Frontiers in oncology 9:1147 DOI 10.3389/fonc.2019.01147.

Imran M, Salehi B, Sharifi-Rad J, Aslam Gondal T, Saeed F, Imran A, Shahbaz M, Tsouh Fokou PV, Umair Arshad M, Khan H, Guerreiro SG, Martins N, Estevinho LM. 2019. Kaempferol: A Key Emphasis to Its Anticancer Potential. Molecules : a journal of synthetic chemistry and natural product chemistry 24: DOI 10.3390/molecules24122277.

Kaneuchi M, Sasaki M, Tanaka Y, Sakuragi N, Fujimoto S, Dahiya R. 2003. Quercetin regulates growth of Ishikawa cells through the suppression of EGF and cyclin D1. International journal of oncology 22:159-164.

Lei X, Guo J, Wang Y, Cui J, Feng B, Su Y, Zhao H, Yang W, Hu Y. 2019. Inhibition of endometrial carcinoma by Kaempferol is interceded through apoptosis induction, G2/M phase cell cycle arrest, suppression of cell invasion and upregulation of m-TOR/PI3K signalling pathway. Journal of B.U.ON. : official journal of the Balkan Union of Oncology 24:1555-1561.

Li H, Ji HS, Kang JH, Shin DH, Park HY, Choi MS, Lee CH, Lee IK, Yun BS, Jeong TS. 2015. Soy Leaf Extract Containing Kaempferol Glycosides and Pheophorbides Improves Glucose Homeostasis by Enhancing Pancreatic $\beta$-Cell Function and Suppressing Hepatic Lipid Accumulation in $\mathrm{db} / \mathrm{db}$ Mice. Journal of agricultural and food chemistry 63:71987210 DOI 10.1021/acs.jafc.5b01639.

Li W, Song K, Wang S, Zhang C, Zhuang M, Wang Y, Liu T. 2019. Anti-tumor potential of astragalus polysaccharides on breast cancer cell line mediated by macrophage activation. Materials science \& engineering. C, Materials for biological applications 98:685-695

Peer) reviewing PDF | (2021:02:58138:2:0:NEW 17 Jul 2021) 
561

562

563

564

565

566

567

568

569

570

571

572

573

574

575

576

577

578

579

580

581

582

583

584

585

586

587

588

589

590

591

592

593

594

595

596

DOI 10.1016/j.msec.2019.01.025.

Liao H, Hu L, Cheng X, Wang X, Li J, Banbury L, Li R. 2017. Are the Therapeutic Effects of Huangqi (Astragalus membranaceus) on Diabetic Nephropathy Correlated with Its Regulation of Macrophage iNOS Activity. Journal of immunology research 2017:3780572 DOI 10.1155/2017/3780572.

Lin TH, Hsu WH, Tsai PH, Huang YT, Lin CW, Chen KC, Tsai IH, Kandaswami CC, Huang CJ, Chang GD, Lee MT, Cheng CH. 2017. Dietary flavonoids, luteolin and quercetin, inhibit invasion of cervical cancer by reduction of UBE2S through epithelialmesenchymal transition signaling. Food \& function 8:1558-1568 DOI 10.1039/c6fo00551a.

Liu C, Wang K, Zhuang J, Gao C, Li H, Liu L, Feng F, Zhou C, Yao K, Deng L, Wang L, Li J, Sun C. 2019a. The Modulatory Properties of Astragalus membranaceus Treatment on Triple-Negative Breast Cancer: An Integrated Pharmacological Method. Frontiers in pharmacology 10:1171 DOI 10.3389/fphar.2019.01171.

Liu J, Nile SH, Xu G, Wang Y, Kai G. 2019c. Systematic exploration of Astragalus membranaceus and Panax ginseng as immune regulators: Insights from the comparative biological and computational analysis. Phytomedicine : international journal of phytotherapy and phytopharmacology 153077 DOI 10.1016/j.phymed.2019.153077.

Liu J, Zhou S, Li S, Jiang Y, Wan Y, Ma X, Cheng W. 2019b. Eleven genes associated with progression and prognosis of endometrial cancer (EC) identified by comprehensive bioinformatics analysis. Cancer cell international 19:136 DOI 10.1186/s12935-0190859-1.

Maag J. 2018. gganatogram: An R package for modular visualisation of anatograms and tissues based on ggplot2. F1000Research 7:1576 DOI 10.12688/f1000research.16409.2.

Martin A, Ochagavia ME, Rabasa LC, Miranda J, Fernandez-de-Cossio J, Bringas R. 2010. BisoGenet: a new tool for gene network building, visualization and analysis. $B M C$ bioinformatics 11:91 DOI 10.1186/1471-2105-11-91.

Missiuro PV, Liu K, Zou L, Ross BC, Zhao G, Liu JS, Ge H. 2009. Information flow analysis of interactome networks. PLoS computational biology 5:e1000350 DOI 10.1371/journal.pcbi.1000350.

Ong S, Shanmugam MK, Fan L, Fraser SE, Arfuso F, Ahn KS, Sethi G, Bishayee A. 2019. Focus on Formononetin: Anticancer Potential and Molecular Targets. Cancers 11: DOI 10.3390/cancers 11050611 .

Pappa KI, Polyzos A, Jacob-Hirsch J, Amariglio N, Vlachos GD, Loutradis D, Anagnou NP. 2015. Profiling of Discrete Gynecological Cancers Reveals Novel Transcriptional Modules and Common Features Shared by Other Cancer Types and Embryonic Stem 
Cells. PloS one 10:e142229 DOI 10.1371/journal.pone.0142229.

Park S, Bazer FW, Lim W, Song G. 2018. The O-methylated isoflavone, formononetin, inhibits human ovarian cancer cell proliferation by sub G0/G1 cell phase arrest through PI3K/AKT and ERK1/2 inactivation. Journal of cellular biochemistry 119:7377-7387 DOI $10.1002 /$ jcb.27041.

Raman K, Damaraju N, Joshi GK. 2014. The organisational structure of protein networks: revisiting the centrality-lethality hypothesis. Systems and synthetic biology 8:73-81 DOI 10.1007/s11693-013-9123-5.

Repana D, Nulsen J, Dressler L, Bortolomeazzi M, Venkata SK, Tourna A, Yakovleva A, Palmieri T, Ciccarelli FD. 2019. The Network of Cancer Genes (NCG): a comprehensive catalogue of known and candidate cancer genes from cancer sequencing screens. Genome biology 20:1 DOI 10.1186/s13059-018-1612-0.

Ru J, Li P, Wang J, Zhou W, Li B, Huang C, Li P, Guo Z, Tao W, Yang Y, Xu X, Li Y, Wang Y, Yang L. 2014. TCMSP: a database of systems pharmacology for drug discovery from herbal medicines. Journal of cheminformatics 6:13 DOI 10.1186/17582946-6-13.

Sakuragi N, Salah-eldin AE, Watari H, Itoh T, Inoue S, Moriuchi T, Fujimoto S. 2002. Bax, Bcl-2, and p53 expression in endometrial cancer. Gynecologic oncology 86:288-296 DOI 10.1006/gyno.2002.6742.

Scambia G, Ranelletti FO, Benedetti Panici P, Piantelli M, Bonanno G, De Vincenzo R, Ferrandina G, Maggiano N, Capelli A, Mancuso S. 1992. Inhibitory effect of quercetin on primary ovarian and endometrial cancers and synergistic activity with cisdiamminedichloroplatinum (II). Gynecologic oncology 45:13-19 DOI 10.1016/00908258(92)90484-z.

Shan H, Zheng X, Li M. 2019. The effects of Astragalus Membranaceus Active Extracts on Autophagy-related Diseases. International journal of molecular sciences 20: DOI 10.3390/ijms20081904.

Sidders B, Karlsson A, Kitching L, Torella R, Karila P, Phelan A. 2018. Network-Based Drug Discovery: Coupling Network Pharmacology with Phenotypic Screening for Neuronal Excitability. Journal of molecular biology 430:3005-3015 DOI 10.1016/j.jmb.2018.07.016.

Siegel RL, Miller KD, Jemal A. 2020. Cancer statistics, 2020. CA: a cancer journal for clinicians 70:7-30 DOI 10.3322/caac.21590.

Stine ZE, Walton ZE, Altman BJ, Hsieh AL, Dang CV. 2015. MYC, Metabolism, and Cancer. Cancer discovery 5:1024-1039 DOI 10.1158/2159-8290.CD-15-0507. 
632

633

634

635

636

637

638

639

640

641

642

643

644

645

646

647

648

649

650

651

652

653

654

655

656

657

658

659

660

661

662

663

664

665

666

667

Suen AA, Jefferson WN, Wood CE, Williams CJ. 2019. SIX1 Regulates Aberrant Endometrial Epithelial Cell Differentiation and Cancer Latency Following Developmental Estrogenic Chemical Exposure. Molecular cancer research : MCR 17:2369-2382 DOI 10.1158/1541-7786.MCR-19-0475.

Tamura R, Yoshihara K, Yamawaki K, Suda K, Ishiguro T, Adachi S, Okuda S, Inoue I, Verhaak RG, Enomoto T. 2015. Novel kinase fusion transcripts found in endometrial cancer. Scientific reports 5:18657 DOI 10.1038/srep18657.

Tang Y, Li M, Wang J, Pan Y, Wu FX. 2015. CytoNCA: a cytoscape plugin for centrality analysis and evaluation of protein interaction networks. Bio Systems 127:67-72 DOI 10.1016/j.biosystems.2014.11.005.

Urick ME, Bell DW. 2019. Clinical actionability of molecular targets in endometrial cancer. Nature reviews. Cancer 19:510-521 DOI 10.1038/s41568-019-0177-x.

Wang L, Lee IM, Zhang SM, Blumberg JB, Buring JE, Sesso HD. 2009. Dietary intake of selected flavonols, flavones, and flavonoid-rich foods and risk of cancer in middle-aged and older women. The American journal of clinical nutrition 89:905-912 DOI 10.3945/ajen.2008.26913.

Wang X, Yang Y, An Y, Fang G. 2019. The mechanism of anticancer action and potential clinical use of kaempferol in the treatment of breast cancer. Biomedicine \& pharmacotherapy $=$ Biomédecine \& pharmacothérapie 117:109086 DOI 10.1016/j.biopha.2019.109086.

Wang Y, Zhou R, Wang J. 2019. Relationship between Hypothyroidism and Endometrial Cancer. Aging and disease 10:190-196 DOI 10.14336/AD.2018.0224.

Webb PM, Na R, Weiderpass E, Adami HO, Anderson KE, Bertrand KA, Botteri E, Brasky TM, Brinton LA, Chen C, Doherty JA, Lu L, McCann SE, Moysich KB, Olson S, Petruzella S, Palmer JR, Prizment AE, Schairer C, Setiawan VW, Spurdle AB, Trabert B, Wentzensen N, Wilkens L, Yang HP, Yu H, Risch HA, Jordan SJ. 2019. Use of aspirin, other nonsteroidal anti-inflammatory drugs and acetaminophen and risk of endometrial cancer: the Epidemiology of Endometrial Cancer Consortium. Annals of oncology : official journal of the European Society for Medical Oncology 30:310-316 DOI 10.1093/annonc/mdy541.

Wu Y, Zhang X, Li Z, Yan H, Qin J, Li T. 2017. Formononetin inhibits human bladder cancer cell proliferation and invasiveness via regulation of miR-21 and PTEN. Food \& function 8:1061-1066 DOI 10.1039/c6fo01535b.

Xia C, He Z, Cai Y. 2020. Quantitative proteomics analysis of differentially expressed proteins induced by astragaloside IV in cervical cancer cell invasion. Cellular \& molecular biology letters 25:25 DOI 10.1186/s11658-020-00218-9. 
668

669

670

671

672

673

674

675

676

677

678

679

680

681

682

683

684

685

686

687

688

689

690

691

692

693

694

695

696

697

698

699

700

Xiaoming O, Cheng Z, Yun Z, Degui L, Miaoling L. 2016. Effects of astragalus injection on the expression of $\beta$-Catenin and E-cadherin in human endometrial carcinoma cells. Journal of Molecular Imaging 39:292-296 DOI 10.3969/j.issn.1674-4500.2016.03.28.

Xie JH, Jin ML, Morris GA, Zha XQ, Chen HQ, Yi Y, Li JE, Wang ZJ, Gao J, Nie SP, Shang P, Xie MY. 2016. Advances on Bioactive Polysaccharides from Medicinal Plants. Critical reviews in food science and nutrition 56 Suppl 1:S60-84 DOI 10.1080/10408398.2015.1069255.

Xin M, Wang Y, Ren Q, Guo Y. 2019. Formononetin and metformin act synergistically to inhibit growth of MCF-7 breast cancer cells in vitro. Biomedicine \& pharmacotherapy = Biomédecine \& pharmacothérapie 109:2084-2089 DOI 10.1016/j.biopha.2018.09.033.

Xiyao H, Chao W, Lihong G. 2019. Effects of Huangqi Fuzheng Tang on the Expressions of Serum NGAL and NGALR Protein and Bone Marrow Arresting in Patients Undergoing Chemotherapy. Western Journal of Traditional Chinese Medicine 32:1-4.

Yufang K, Yan D. 2019. Clinical Study on Huangqi Injection and Cis-platinum Complexes in Treathing Endometrial Carcinoma. Western Journal of Traditional Chinese Medicine 32:86-89.

Zhang J, Liu L, Wang J, Ren B, Zhang L, Li W. 2018. Formononetin, an isoflavone from Astragalus membranaceus inhibits proliferation and metastasis of ovarian cancer cells. Journal of ethnopharmacology 221:91-99 DOI 10.1016/j.jep.2018.04.014.

Zhang RZ, Yu SJ, Bai H, Ning K. 2017. TCM-Mesh: The database and analytical system for network pharmacology analysis for TCM preparations. Scientific reports 7:2821 DOI 10.1038/s41598-017-03039-7.

Zhang W, Tao Q, Guo Z, Fu Y, Chen X, Shar PA, Shahen M, Zhu J, Xue J, Bai Y, Wu Z, Wang Z, Xiao W, Wang Y. 2016. Systems Pharmacology Dissection of the Integrated Treatment for Cardiovascular and Gastrointestinal Disorders by Traditional Chinese Medicine. Scientific reports 6:32400 DOI 10.1038/srep32400.

Zhang X, Zhang W, Jiang Y, Liu K, Ran L, Song F. 2019. Identification of functional lncRNAs in gastric cancer by integrative analysis of GEO and TCGA data. Journal of cellular biochemistry 120:17898-17911 DOI 10.1002/jcb.29058.

Zhang Y, Chen C, Zhang J. 2019. Effects and significance of formononetin on expression levels of HIF-1 a and VEGF in mouse cervical cancer tissue. Oncology letters 18:22482253 DOI 10.3892/ol.2019.10567.

Peer) reviewing PDF | (2021:02:58138:2:0:NEW 17 Jul 2021) 


\section{Figure 1}

Figure 1. Volcanic map and heatmap of 360 DEGs (including 204 up-regulated genes and 156 down-regulated genes) from GSE63678.

(A) Volcano map of all genes. Pink nodes represent DEGs with $\log F C>1$ and P-value $<0.05$. Blue nodes represent DEGs with $\log F C<-1$ and p-values $<0.05$. (B) Top 40 differentially expressed genes between normal and EC tissues. Each column represents a sample, and each row represents one gene. The gradual colour changes from blue to pink represent the gene expression changing from downregulation to upregulation. C: genes from normal tissues; T: genes from EC tissues.
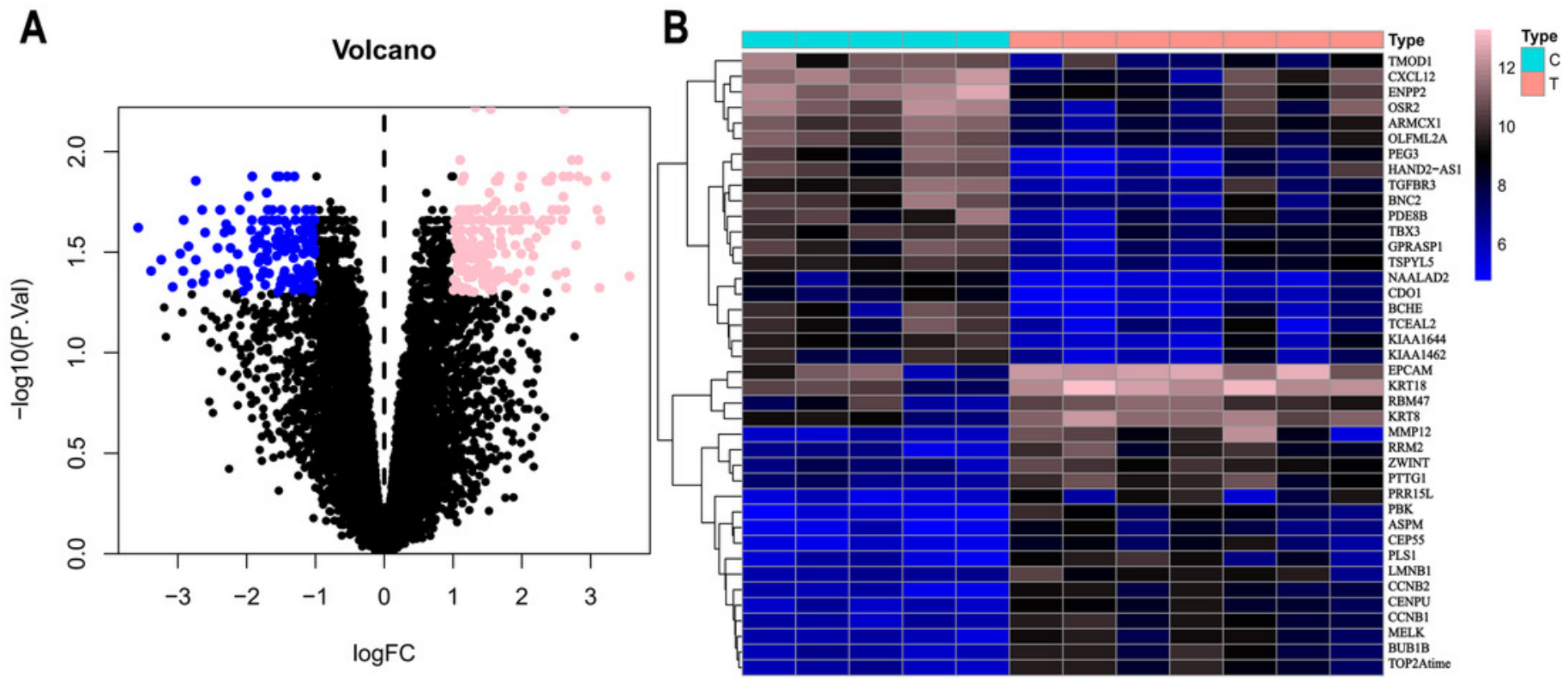


\section{Figure 2}

Figure 2. Volcanic map and heatmap of 11531 DEGs (including 7727 up-regulated genes and 3804 down-regulated genes) from TCGA.

(A) Red and blue plots: differentially expressed mRNAs; black plots: normally expressed mRNAs. (B) Red: upregulation; blue: downregulation.

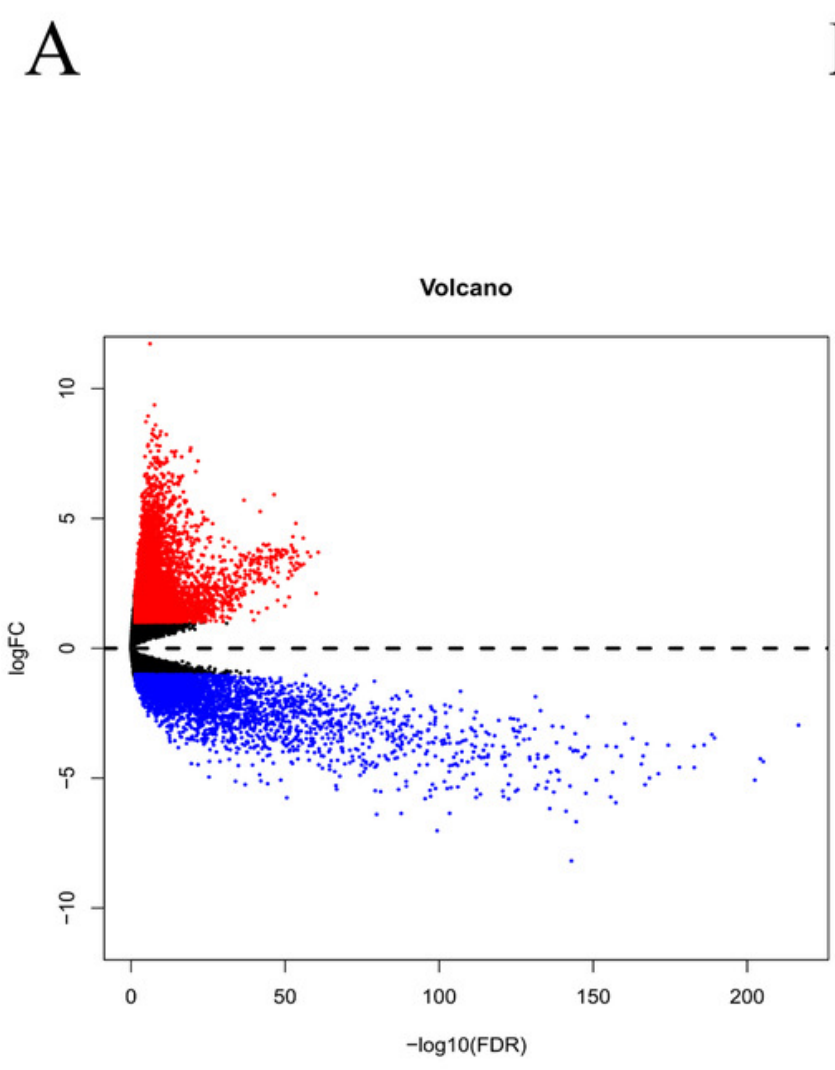

B
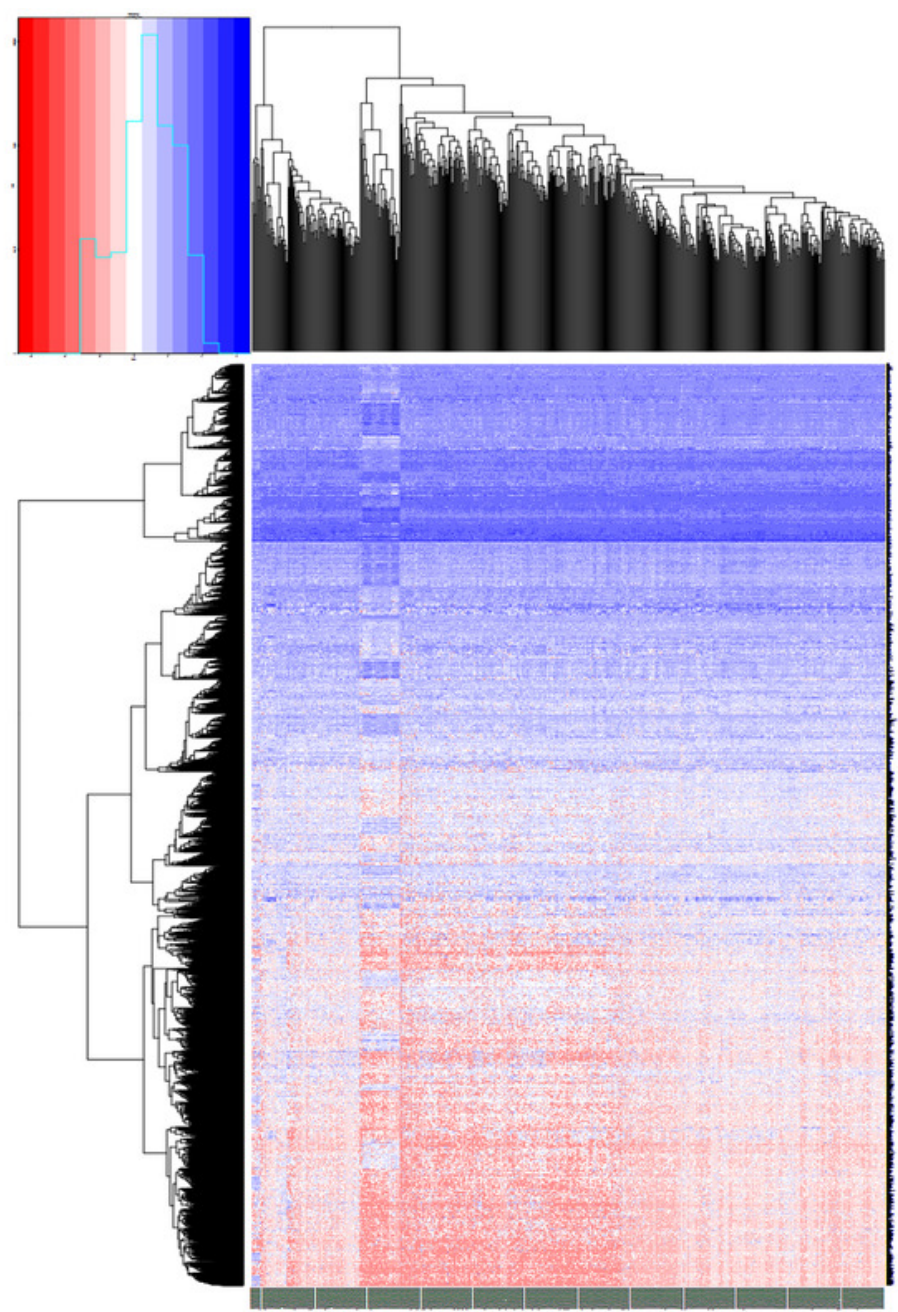
Figure 3

Figure 3. Venn diagram shows the intersecting DEGs from GEO and TCGA.

Blue area: GEO dataset; orange area: TCGA dataset; cross area: DEGs expressed in both databases. 


\section{GEO dataset}

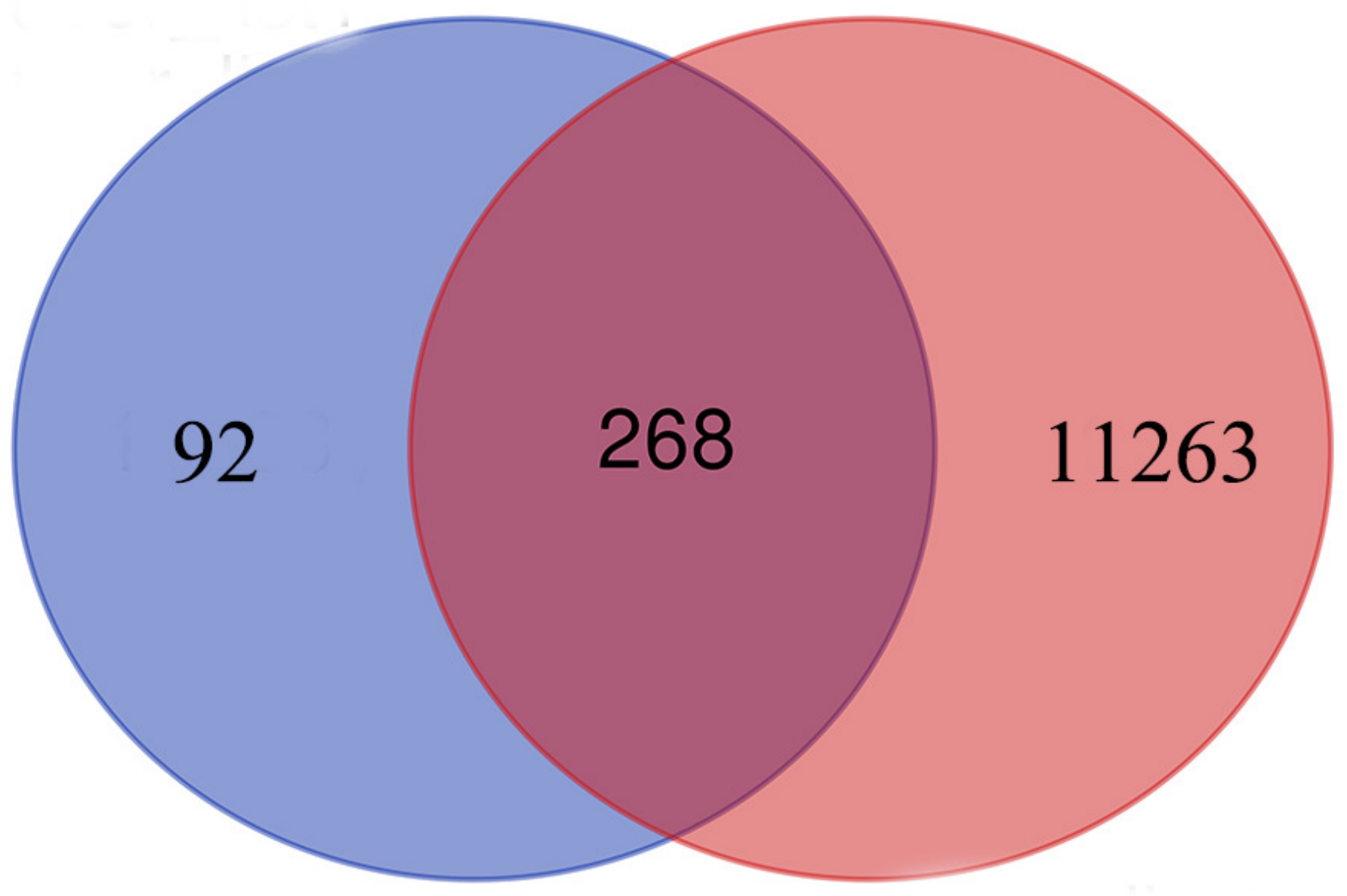

TCGA dataset 


\section{Figure 4}

Figure 4. Network of active compounds and common targets based on Cytoscape 3.7.1. (https://cytoscape.org/).

Pink ovals represent active compounds of Astragalus membranaceus. The yellow triangles in the middle represent the targets. The grey lines indicate the relationship between compounds and targets. 


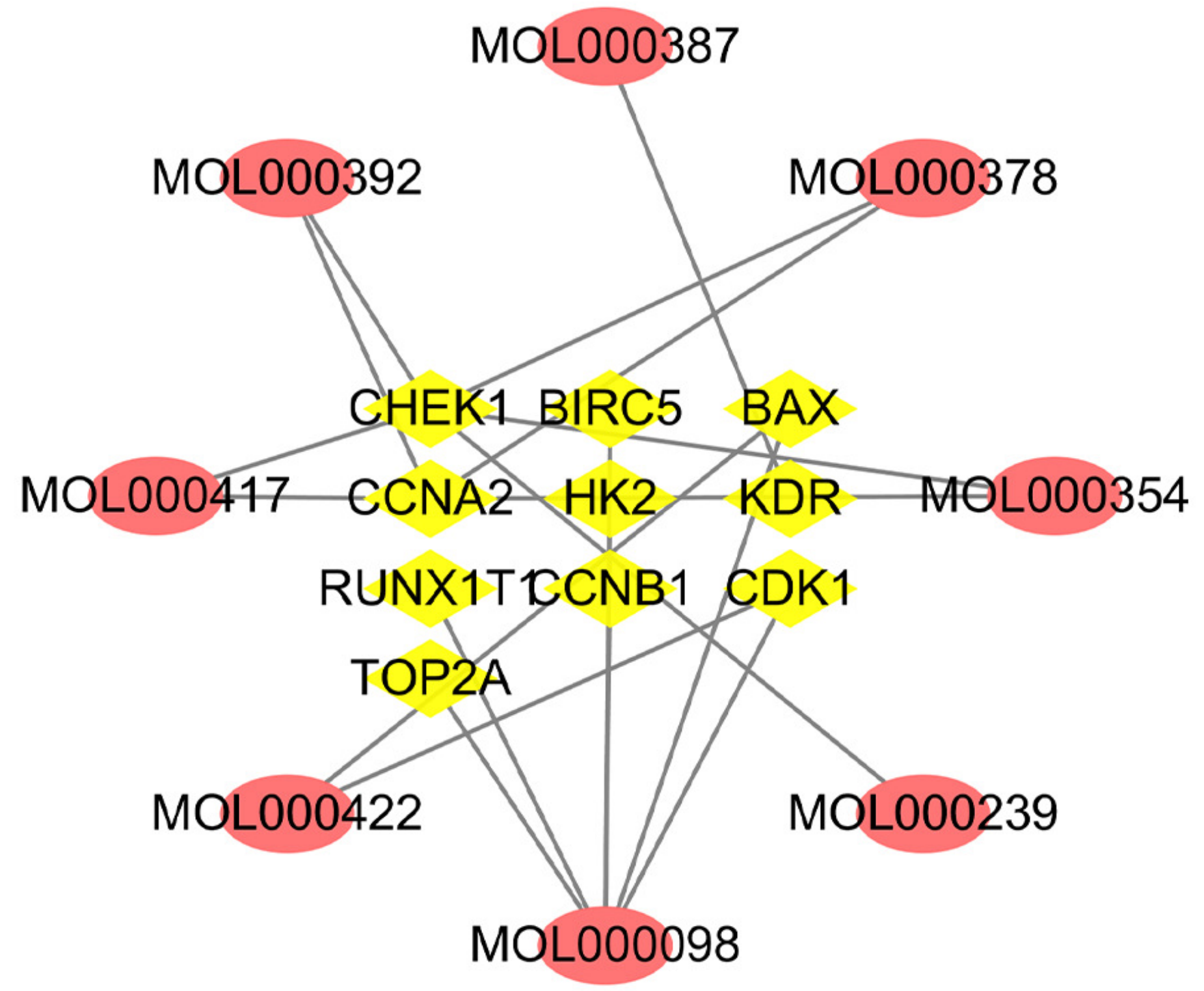


Figure 5

Figure 5. The process for constructing the core PPI network of the 13 targets.

(A) The original PPI network. (B) The PPI network when DC > 61. (C) The core PPI network when $\mathrm{DC}>61$ and $\mathrm{BC}>100$.

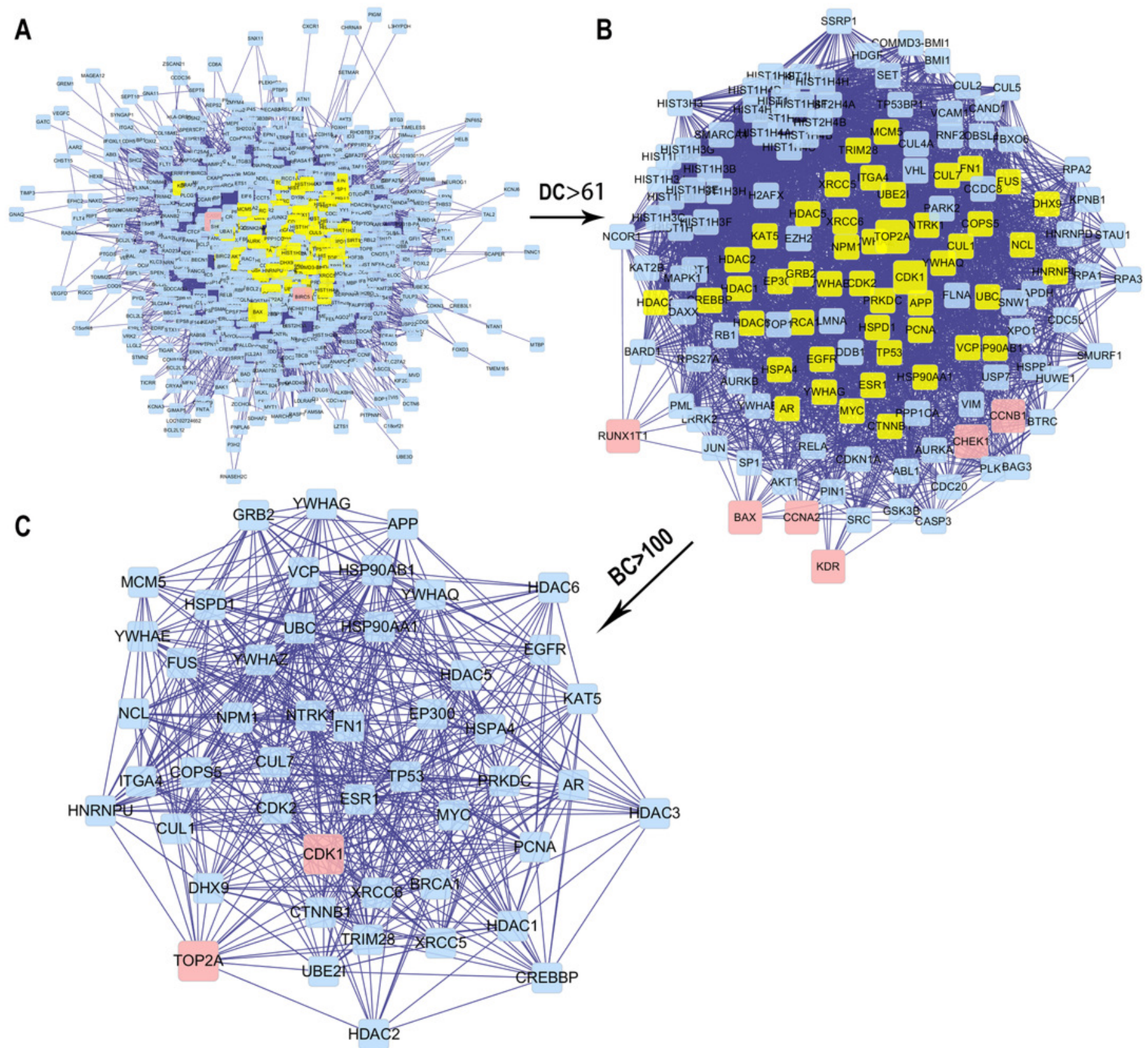




\section{Figure 6}

Figure 6. GO and KEGG pathway analyses of 13 target genes.

\section{(A) Biological process category. (B) Cellular component category. (C) Molecular function}

category. (D) KEGG pathway analysis. The cut-off criterion for a significant difference was $\mathrm{P}<$ 0.05 .

A DNA integrity checkpoint signal transduction involved in DNA damage checkpoint signal transduction involved in cell cycle checkpoint organelle fission. cell cycle checkpoint mitotic DNA integrity checkpoint negative regulation of mitotic cell cycle signal transduction in response to DNA damage DNA damage checkpoint mitotic cell cycle checkpoint nuclear division chromosome condensation. DNA damage response, signal transduction by 553 class mediator resulting in cell cycle arrest signal transduction involved in mitotic G1 DNA damage checkpoint

intracellular siznal transduction involved in G1 DVA damage checkpoint.
signal transduction involved in mitotic cell cycle checkpoint. signal transduction involved in mitotic DNA damage checkpoint. signal transduction involved in mitotic DNA integrity checkpoint

C

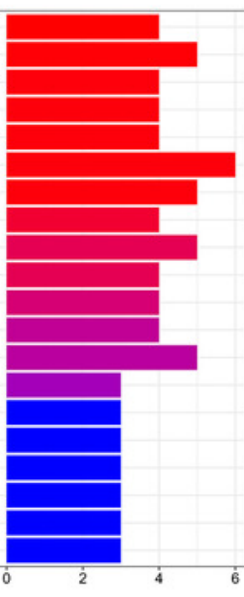

B

D

histone kinase activity heat shock protein binding .

Hsp70 protein binding cyclin-dependent protein serine/ threonine kinase regulator activity chaperone binding

protein kinase regulator activity kinase regulator activity glucose binding BH domain binding .

death domain binding RNA polymerase II CTD heptapeptide repeat kinase activity DNA binding, bending

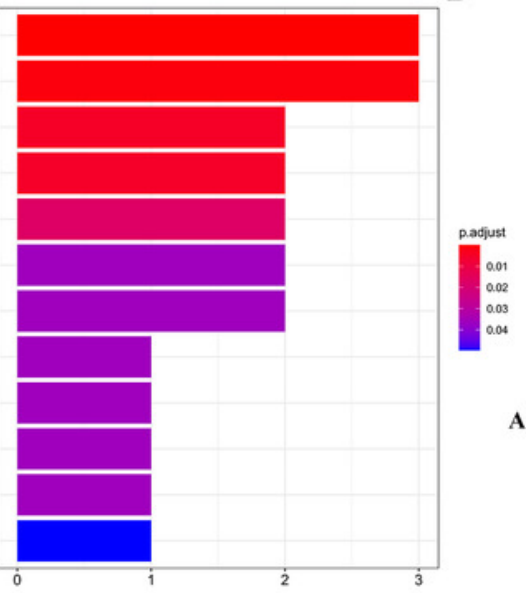

cyclin-dependent protein kinase holoenzyme complex
condensed chromosome serine/threonine protein kinase complex protein kinase complex chromosomal region chromosomal region
transferase complex, transferring spindle microtubule spindle condensed nuclear chromosome so-05 condensed chromosome kinetochore condensed chromosome, centromeric region mic region
midbody

mitochondrial outer membrane chromosome, centromeric region organelle outer membrane outer membrane host intracellular part host intracellular region

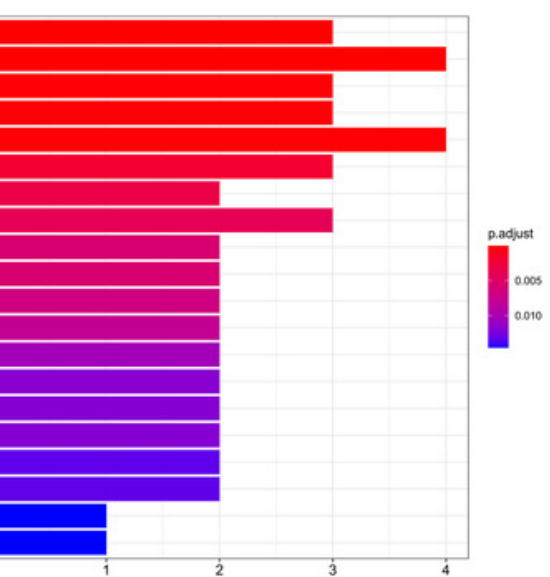

P53 signalling pathway Cell cycle

Cellular senescence

Viral carcinogenesis Human immunodeficiency virus 1 infection Platinum drug resistance Progesterone-mediated oocyte maturation Hepatitis B

Transeriptional misregulation Human T-cell leukemia poptosis - multiple 1 infection Acute myeloid leukemia. EGFR tyrosine kinase inhibitor resistance Endometrial cancer -

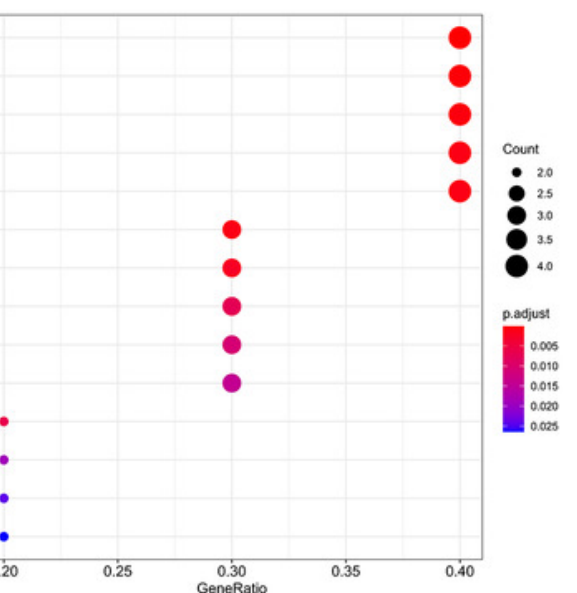


Figure 7

Figure 7. Pathway-targets analysis.

The red triangle represents the KEGG pathway. The orange square represents the related targets. 


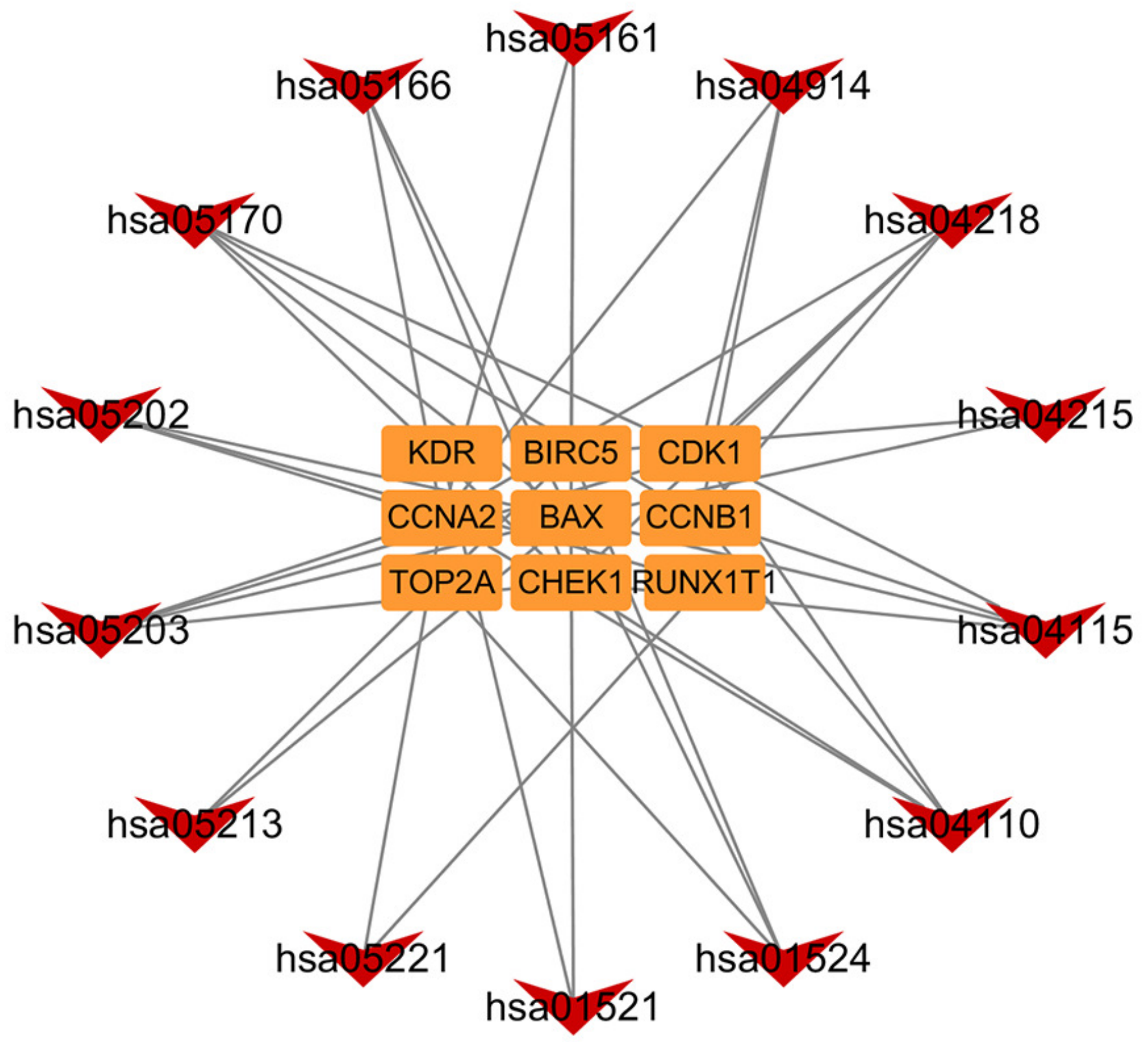


Figure 8

Cell viability assay (A) and Colony formation assay (B) detect the effects of different concentrations of formononetin on the proliferation of endometrial cancer cell lines. $* * * P<0.001$. 


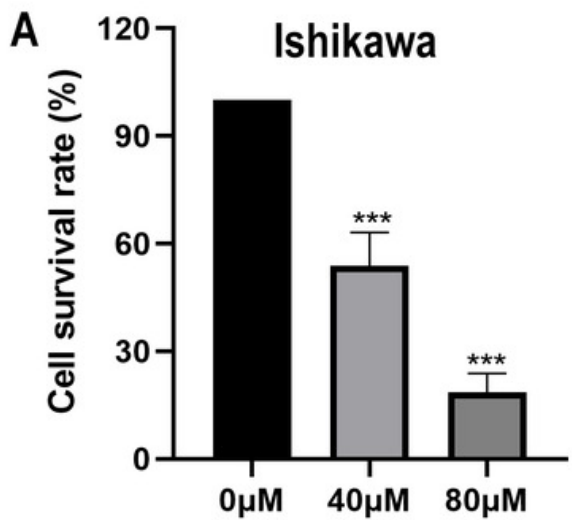

Formononetin

B
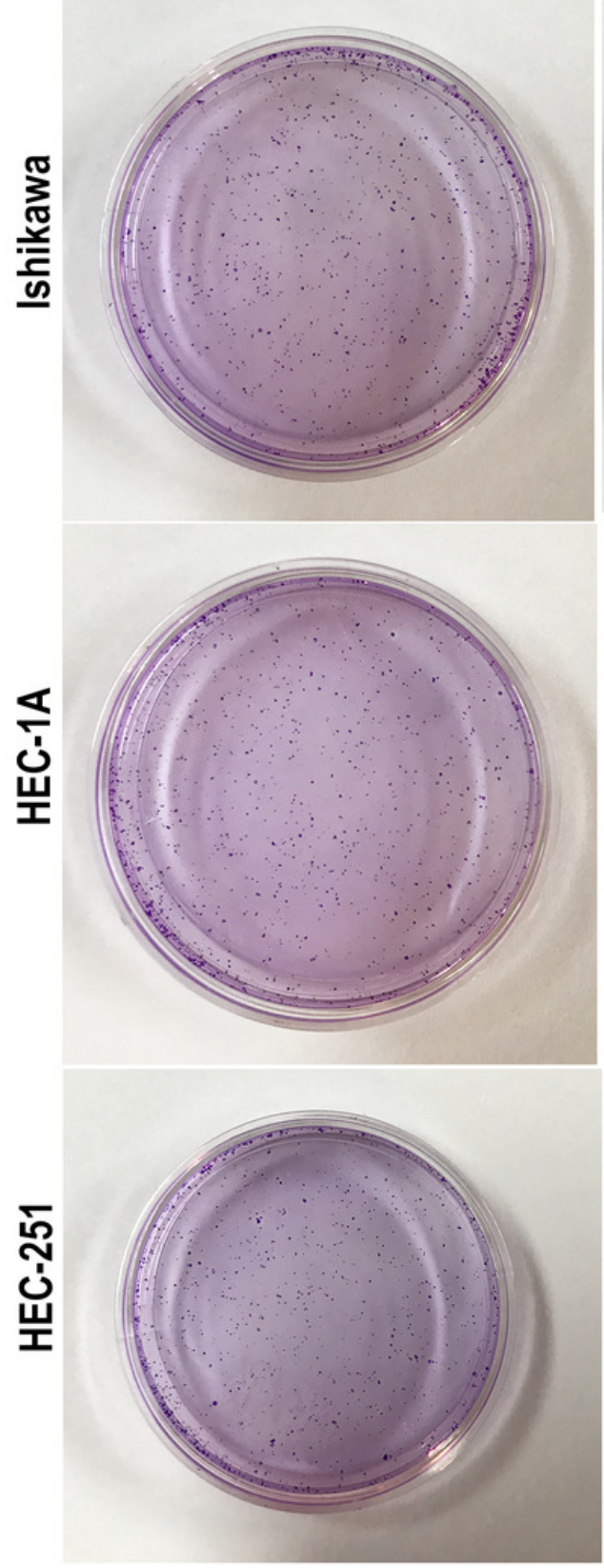

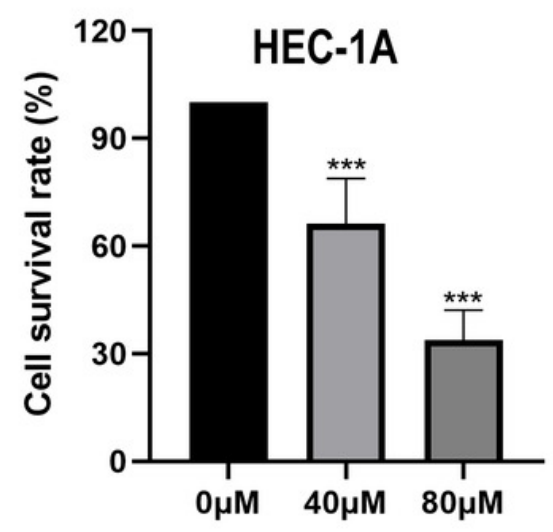

Formononetin

$40 \mu \mathrm{M}$
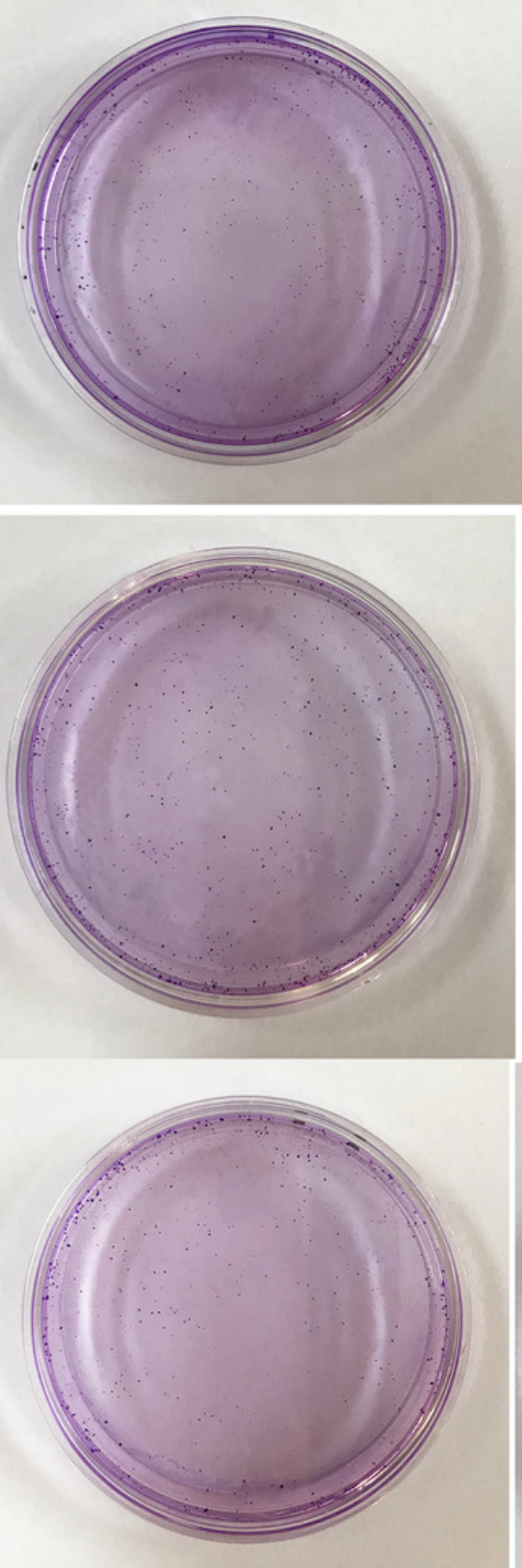
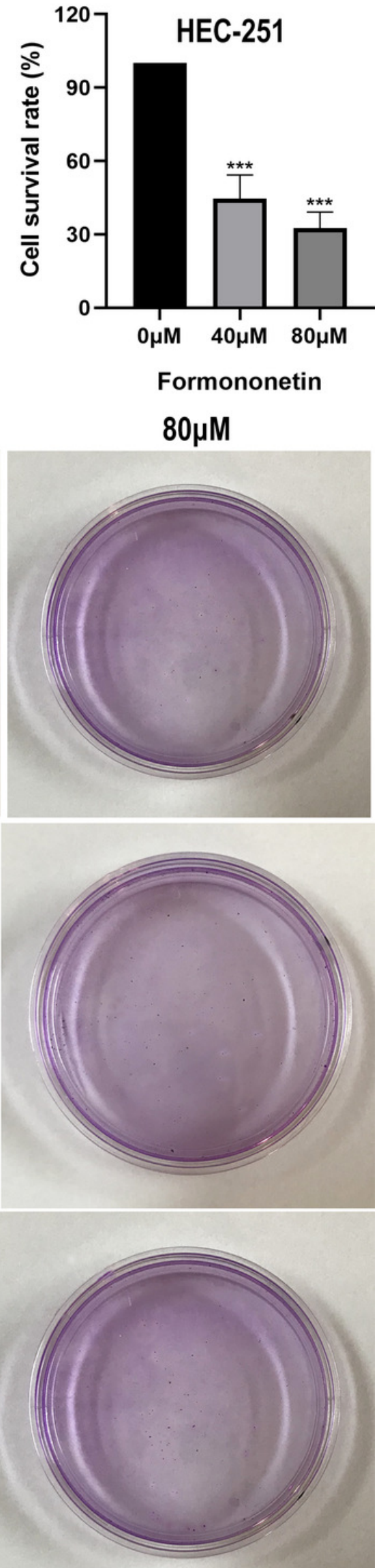
Figure 9

Figure 9. The qPCR assay and Western blot assay were used to explore the differences in $E R \beta$ and $p 53$ expression when formononetin exerts an anti-tumour effect.

Relative expression of (A) ER $\beta$ and (B) p53 in endometrial cancer cell lines by qPCR assay. (C) Protein expression levels of ER $\beta$ and p53 in endometrial cancer cell lines by western blot assay. ${ }^{* * * P}<0.001$ 


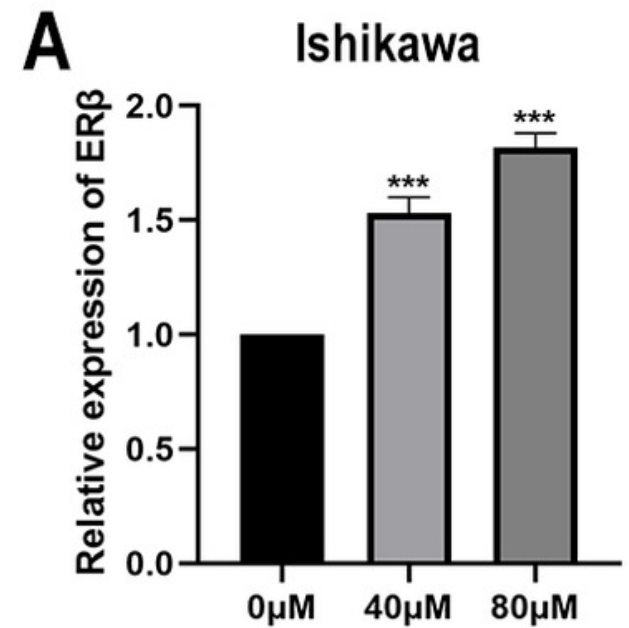

B

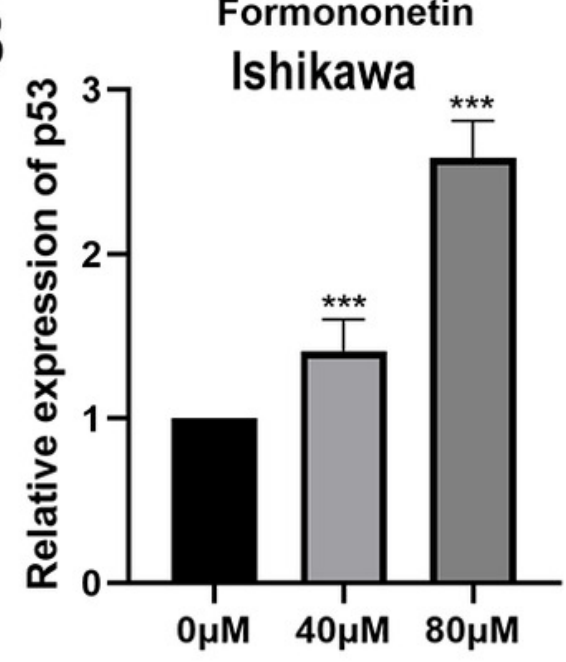

Formononetin

C

Ishikawa

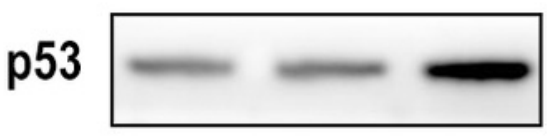

ER $\beta$

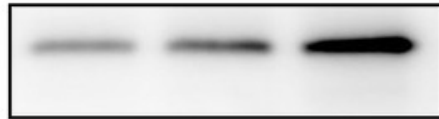

$\beta$-actin

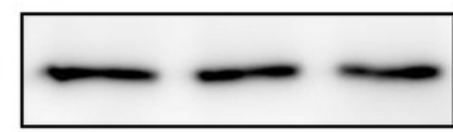

$0 \mu \mathrm{M} \quad 40 \mu \mathrm{M} \quad 80 \mu \mathrm{M}$ Formononetin
HEC-1A

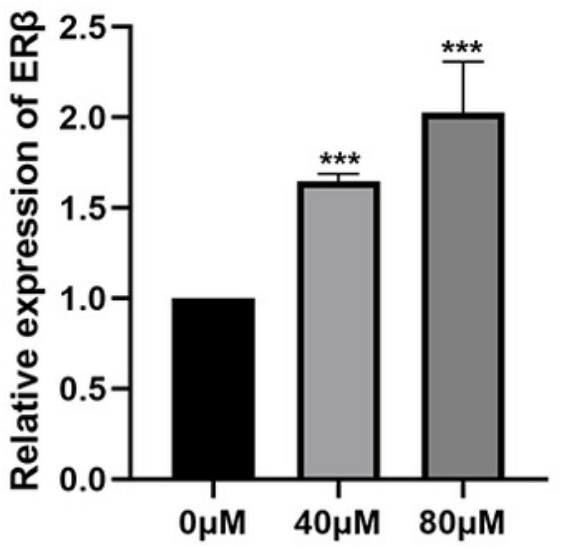

Formononetin

HEC-1A

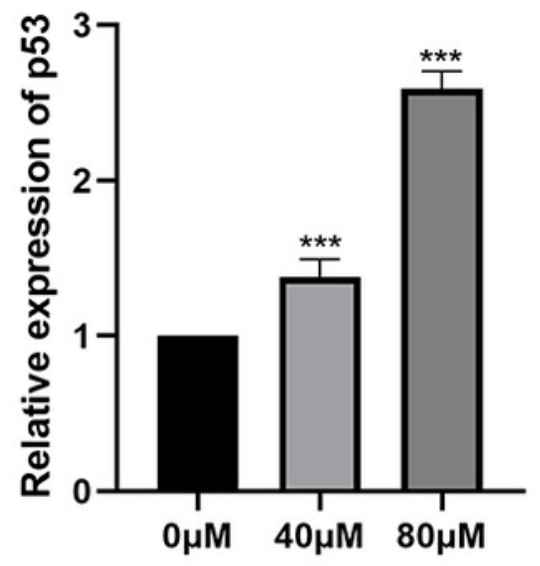

Formononetin

HEC-1A
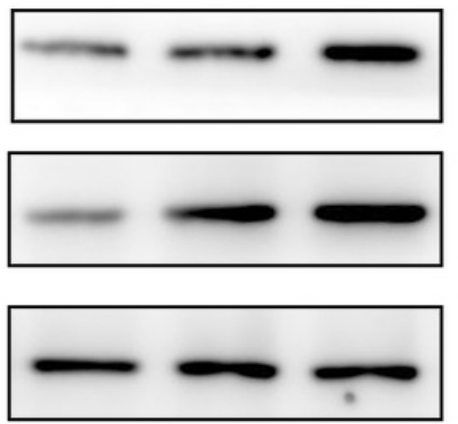

$0 \mu \mathrm{M} \quad 40 \mu \mathrm{M} \quad 80 \mu \mathrm{M}$ Formononetin
HEC-251

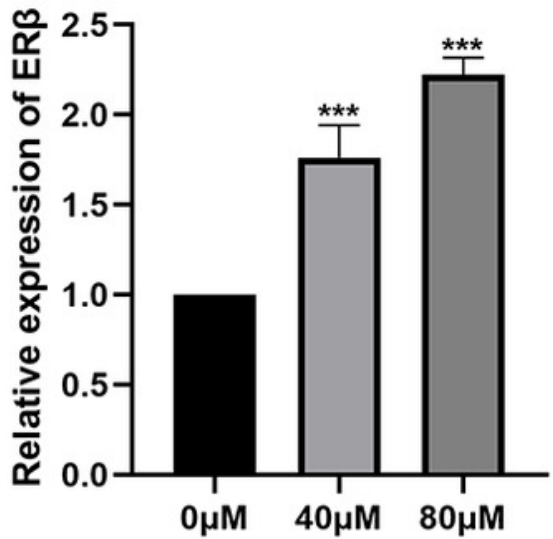

Formononetin

HEC-251

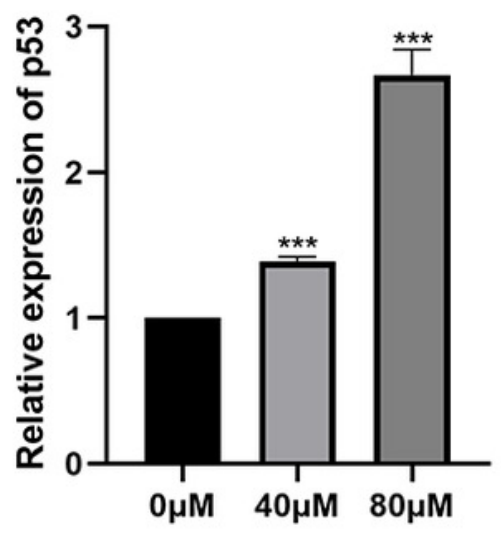

Formononetin

HEC-251
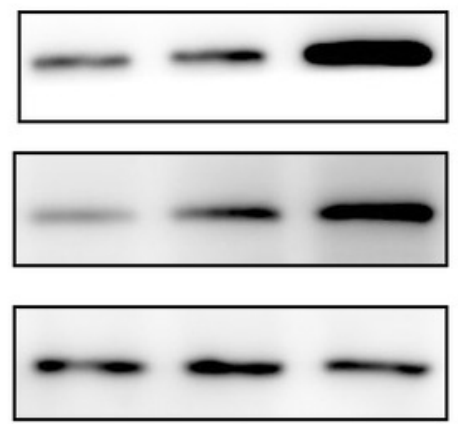

$0 \mu \mathrm{M} \quad 40 \mu \mathrm{M} \quad 80 \mu \mathrm{M}$ Formononetin 


\section{Table $\mathbf{1}$ (on next page)}

Table 1: Top ten compounds in Astragalus membranaceus and the targets 
1 Table 1: Top ten compounds in Astragalus membranaceus and the targets

2

\begin{tabular}{lll}
\hline MolId & MolName & Target \\
MOL000211 & Mairin & Progesterone receptor \\
MOL000239 & Jaranol & $\begin{array}{l}\text { Nitric oxide synthase, } \\
\text { inducible }\end{array}$ \\
MOL000296 & Hederagenin & Progesterone receptor \\
MOL000354 & Isorhamnetin & Nitric oxide synthase, inducible \\
MOL000371 & 3,9-di-O-methylnissolin & Nitric oxide synthase, inducible \\
MOL000378 & 7-O-methylisomucronulatol & Nitric oxide synthase, inducible \\
MOL000379 & $\begin{array}{l}\text { 9,10-dimethoxypterocarpan-3-O-tail-D- } \\
\text { glucoside }\end{array}$ & Prostaglandin G/H synthase 2 \\
& $\begin{array}{l}\text { (6aR,11aR)-9,10-dimethoxy-6a,11a-dihydro- } \\
\text { 6H-benzofurano[3,2-c]chromen-3-ol }\end{array}$ & Nitric oxide synthase, inducible \\
MOL000380 & Pifendate & Prostaglandin G/H synthase 2 \\
MOL000387 & Formononetin & Nitric oxide synthase, inducible \\
MOL00392 & For &
\end{tabular}




\section{Table 2 (on next page)}

Table 2: Common target genes and corresponding active components of endometrial cancer and Astragalus membranaceus 
1 Table 2: Common target genes and corresponding active components of endometrial cancer and

2 Astragalus membranaceus

\begin{tabular}{ccc}
\hline MolId & MolName & Common target genes \\
\hline MOL000239 & Jaranol & CHEK1 \\
MOL000387 & Bifendate & KDR \\
MOL000354 & Isorhamnetin & \\
MOL000417 & Calycosin & \\
MOL000378 & 7-O-methylisomucronulatol & \\
MOL000392 & Formononetin & \\
MOL000422 & Kaempferol & BAX, CDK1 \\
& & BAX, RUNX1T1, \\
MOL000098 & Quercetin & CDK1, BIRC5, \\
& & CCNB1, TOP2A, HK2 \\
\hline
\end{tabular}

3 